\title{
A novel hybrid bacteria-chemotaxis spiral-dynamic algorithm with application to modelling of flexible systems
}

\author{
A. N. K. Nasir ${ }^{[1]}$ and M. O. Tokhi ${ }^{[2]}$. \\ ${ }^{[1]}$ Faculty of Electrical \& Electronics Engineering, Universiti Malaysia Pahang, Pekan Pahang \\ Malaysia. \\ ${ }^{[2]}$ School of Engineering, London South Bank University, 103 Borough Road, London SE1 0AA, UK \\ ${ }^{[1]}$ kasruddin@ump.edu.my, ${ }^{[2]}$ tokhim@1sbu.ac.uk
}

\begin{abstract}
This paper presents a novel hybrid optimisation algorithm namely HBCSD, which synergises a bacterial foraging algorithm (BFA) and spiral dynamics algorithm (SDA). The main objective of this strategy is to develop an algorithm that is capable to reach a global optimum point at the end of the final solution with a faster convergence speed compared to its predecessor algorithms. The BFA is incorporated into the algorithm to act as a global search or exploration phase. The solutions from the exploration phase then feed into SDA, which acts as a local search or exploitation phase. The proposed algorithm is used in dynamic modelling of two types of flexible systems, namely a flexible robot manipulator and a twin rotor system. The results obtained show that the proposed algorithm outperforms its predecessor algorithms in terms of fitness accuracy, convergence speed, and timedomain and frequency-domain dynamic characterisation of the two flexible systems.
\end{abstract}

Keywords- Spiral dynamics; bacteria chemotaxis; system identification; flexible manipulator; twin rotor system.

\section{INTRODUCTION}

This section presents a general overview of metaheuristic algorithms and a general description of the two types of metaheuristic algorithms, including their characteristic features considered in this study. Moreover, a brief description of two types of flexible systems and their status in the current research in the context of modelling and controller design is given. Finally, an overview of the application of various types of optimisation algorithms in modelling of flexible systems is presented.

\subsection{FLEXIBLE SYSTEMS}

The research on flexible systems is increasingly gaining attention from researchers worldwide. The application of such system can be extensively found in various sectors such as in robotics (Gharooni et al., 2001), avionics (Hu, 2009), etc. This is due to the numerous advantages they offer compared to their rigid body counterparts. Two types of commonly used flexible manoeuvring system, namely flexible robot manipulator and twin rotor system (TRS) are considered in this work.

Flexible robot manipulators are used in the manufacturing industry as a tool in the production process. A single-link flexible manipulator is considered in this work. This is a single-input multioutput system comprising rigid and flexible dynamics. Electromechanical actuator at the hub of the system produces rotational motion of rigid body while a flexible beam joining the rigid body and payload produces vibrational motion at the end point of the system. The natural vibrational behaviour of the system poses control challenge in applications where positional accuracy is required. However, the flexible structure of the system exhibits a lot of benefits over its rigid counterpart. Unlike a rigid manipulator, it is lighter in weight, has smaller actuator, better mobility, consumes less power, is less expensive, operates cost-efficiently, has higher payload to robot weight ratio and offers more safety to the user (Ostergaad, 2012; Tokhi et al., 2000).

The TRS used in this work is a laboratory scale flexible manoeuvring system, which mimics a real helicopter in hovering mode. In real world, the application of such a system is mostly found as air transportation. It is considered as a very effective air vehicle as it is capable to take off and land in the vertical direction, which requires less space compared to fixed wing aircraft, rotate its body $360^{\circ}$ easily in hovering mode, fly at low altitude (Raptis et al., 2012). The body construction of the system is very unique and complex. It has vertical and horizontal channels to initiate motion in vertical and horizontal directions respectively. Moreover, interaction between both channels introduces coupling effect and hence produces nonlinearity in its dynamic behaviour. While in hovering mode, motioninduced oscillation causes the system to fluctuate and lose stability. Therefore, an efficient control system is required to operate the system effectively.

Modelling and control of flexible systems are challenging tasks. Their vibrational behaviour and nonlinear characteristics make the modelling of such systems a challenge and lead to very complex 
mathematical models. Through conventional modelling approaches such as partial differential equation (Azad, 1994), finite-difference, finite-element methods (Tokhi and Azad, 1995; Tokhi and Mohamed, 1999), to get a very precise model, many parameters should be taken into account and in many cases, certain assumptions have to be made to simplify the derivation of equation of motion, thus reducing the accuracy of the derived model. From a model-based control point of view, an accurate model of the system is very crucial since the effectiveness of a designed controller is based on the derived model. System identification is an alternative approach to acquire dynamic model of the flexible system based on input-output data from the actual system. The availability of optimisation algorithms and powerful computing technology make this method easy to implement, reliable and more importantly can result in highly accurate models. Linear parametric approach is one of various techniques in system identification to estimate a linear model of a system (Ljung, 1999). In this method, a set of unknown parameters in a predefined structure must be identified. The parameters are set of zeros and poles if the structure is represented by a transfer function or a set of coefficients for a differential equation. The applications of this approach in the engineering field have extensively been reported in the literature (Nino et al., 2007; Tavakolpour et al., 2010). Moreover, the implementation of identification for unknown parameters of a dynamic model of a system in linear parametric modelling can be easily realised using metaheuristic algorithms.

\subsection{OVERVIEW OF THE APPLICATION OF OPTIMISATION ALGORITHMS TO MODELLING OF FLEXIBLE SYSTEMS}

The application of various optimisation algorithms to optimally determine dynamic model of predefined linear and non-linear structures for a real system through system identification approach has been extensively found in the literature. This section gives an overview of the application of the algorithm in the modelling of the flexible robot manipulator and the TRS.

Rovner and Cannon, (1987) and Fujimori et al., (1995) employed recursive least squares (RLS) to optimise the dynamic model of a flexible manipulator system. Yurkovich et al., (1990) proposed an exponential data weighted RLS in comparison to original RLS algorithm to optimise autoregressivemoving-average (ARMA) model. Shaheed and Tokhi, (2002) conducted a study comparing least mean squares (LMS), RLS and genetic algorithm (GA) to optimise parameters of an autoregressivemoving-average model with exogenous inputs (ARMAX) model of flexible manipulator. It was shown that the GA could produce better results than the LMS but it needed more time to complete the whole optimisation process compared to the other two contestants. Liu and Sun, (2001) applied observability range space extraction algorithm to optimise a dynamic model for a single link flexible manipulator. Alam and Tokhi, (2007a) employed particle swarm optimisation (PSO) to optimise the parameters of an ARMA model structure of the flexible manipulator. Md Zain et al., (2009a,b) carried out comparative assessment of RLS, GA and hybrid GA-RLS in the optimisation of ARMA model of a flexible manipulator. The results show that the hybrid type algorithm produced better dynamic model compared to the original algorithms but at the expense of longer computation time. Supriyono and Tokhi, (2012) employed adaptive and original bacterial foraging algorithm (BFA) to optimise autoregressive with exogenous inputs (ARX) dynamic model of structure of the flexible manipulator. The results indicated that the adaptive approach produced more adequate dynamic model for the system but the total computation time for the optimisation process was similar. Yatim et al., (2012) performed a study comparing conventional least square and GA to optimise a linearised model of single link manipulator system which was developed based on finite difference mehod. It was found that the conventional least square predicted better model for a linearised system. In another work, the authors compared the PSO with RLS to optimise the same system and it was found that the PSO performed better than the conventional RLS algorithm (Yatim et al., 2013).

For the TRS, on the other hand, the utilisation of the system identification toolbox of Matlab to optimise an ARMAX model was presented by Ahmad et al., (2001). Aldebrez et al., (2004) employed RLS algorithm to optimise a multi-layer perceptron neural network model. Mat Darus et al., (2004) conducted a study on comparing the performances of GA and conventional RLS to optimise an ARMAX model and it was found that the GA predicted better model as compared to the RLS. Alam and Tokhi, (2007b) performed a comparative assessment of GA and PSO to approximate a linear model for one and two degrees-of-freedom (DOF) TRS. The results showed that the PSO had better accuracy and shorter computation time than the GA. Subudhi and Jena, (2009) hybridised the GA, PSO and differential evolutionary (DE) algorithm with back-propagation (BP) algorithm to optimise a neural network model for one DOF around a pitch axis and the hybrid DE was found with fastest convergence speed. A comparative study was performed by Toha and Tokhi, (2010) where the RLS, real-coded GA and PSO with spread-factor were used to determine parameters of ARMA model. The results indicated that the PSO with spread-factor outperformed the other two algorithms. Omar et al., (2011) applied the hybrid RLS-BP to optimise an adaptive neuro-fuzzy model for the twin-rotor motion in the vertical plane or pitch motion. Toha et al., (2012) employed ant colony algorithm to optimise ARX model of the TRS and the results showed that the estimated model was adequate to 
represent system. It is noted from the above that the performance of metaheuristic algorithm in dealing with the modelling issue of a real system is better than the heuristic or other conventional type algorithms.

\subsection{METAHEURISTIC ALGORITHMS}

Metaheuristic algorithms play an important role and are considered as efficient optimisation tools in solving real world problems in various fields (Neri and Cotta, 2012; Zang et al., 2010). A metaheuristic algorithm is a higher level optimisation algorithm comprising a heuristic approach and iterative process in which the strategy is generally inspired from natural phenomena. Alternatively, metaheuristic can be defined as a process that is iteratively generated to guide a subordinate heuristic by combining intelligently various techniques for globally exploring and locally exploiting a search area and utilising learning strategies to structure information in order to efficiently find an optimum solution (Osman and Laporte,1996). The ease of implementation, ability to solve real world problems in various applications and the capability of producing an optimum and a reliable solution are the advantages and among the reasons they have been continuously received attention from researchers around the world. BFA is a popular and well-known metaheuristic type algorithm while spiral dynamics algorithm (SDA) is a newly developed metaheuristic algorithm that has similar advantages and potential to solve real world problems efficiently. Nevertheless, these two algorithms have limitations and drawbacks which are discussed in the following subsections.

\subsubsection{BACTERIAL FORAGING ALGORITHM}

BFA is a metaheuristic algorithm introduced by Passino, (2002) and it was developed based on the adaptation of foraging strategy of living micro-organism in human intestines, namely Escherichia Coli bacteria. The algorithm consists of three main phases, namely chemotaxis, reproduction, and elimination-dispersal.

From optimisation algorithm point of view, chemotaxis is the most important phase in BFA and it contains a very important parameter that determines the performance of the algorithm both in terms of convergence speed and accuracy. This is the step size of a bacterium to move from one place to another and is represented as $C$. Defining a small value for the step size may lead to high accuracy as the bacterium can reach all the points within the search space. However, the drawback of this strategy is that the bacterium needs more steps to reach a target point and this may lead to slower convergence as well as higher computation time. On the contrary, a large value of $C$ may expedite the convergence speed since the bacterium will move with a large step size and it will need fewer steps to reach a goal point. But, this limits the movement of a bacterium within the search space and the bacterium may not reach the optimum location at all if it is located in a remote area. Moreover, this may introduce oscillation (back and forth movement) about the optimum point. Thus, in the bacteria chemotaxis good exploration is associated with large step size and good exploitation is associated with small step size. Hence, a fixed step size creates imbalance between the exploration and exploitation, and this affects the performance of the algorithm.

Reproduction and elimination-dispersal phases are the second and third phases of BFA respectively. All the three processes are continuous and they are repeated until the end of bacteria life. Overall, the BFA is a very effective way to search for global best solution. However, it is relatively complex, has slower convergence and more computation time might be needed to complete the whole search operation. Since the introduction of BFA, many successful applications of the original and modified versions of BFA to solve real world problems have been reported.

Generally, the modifications of BFA are made through adaptive or hybrid approaches. The adaptive approach involves variation of bacteria step size based on fitness cost, index of iteration or combination of both (Dasgupta et al., 2009; Majhi, 2009). Apart from mathematical relationship, the adaptation of bacteria step size can be realised through an intelligent approach (Mishra, 2005; Venkaiah and Kumar, 2011). Synergising BFA with other metaheuristic algorithms is another effective approach to improve the algorithm performance. This technique is gaining attention from researchers worldwide as the strengths or unique properties of two or more algorithms can be combined to complement one another, and thus reduce/eliminate the shortcomings of the original algorithms. Some of the existing BFA hybrid techniques include BFA-PSO (Biswas et al., (2007a), BFA-DE (Biswas et al., 2007b), BFA-GA (Kim et al., 2007), BFA-nelder mead (Panigrahi and Pandi, 2008), BFA-biogeography based algorithm (Lohokare et al., 2009), BFA-tabu (Panikhom et al., 2010) and BFA-bee colony (Zhong et al., 2011).

\subsubsection{SPIRAL DYNAMICS ALGORITHM}

SDA is a metaheuristic algorithm introduced by Tamura and Yasuda, (2011a) and it is inspired from spiral phenomena in nature such as spiral of galaxy, hurricanes and tornados, geometry of nautilus shell, shape of human fingerprint, etc. The unique property of SDA is that it contains a very powerful 
spiral model, which forms a spiral motion trajectory for search agents in a search space for both exploration and exploitation strategies. The model causes all the agents to be diversified and intensified at the initial and final phases of the search operation respectively. The diversification represents exploration of an optimal solution in a wider space with a large step size while the intensification represents exploitation strategy to search for a highly accurate solution in a remote location with smaller step size. Moreover, with the presence of spiral model, the step size of agents can be steadily controlled and dynamically varied throughout the search operation. Moreover, the motion of all agents is always guided towards the best optimum location found in that particular iteration. All of these features lead the algorithm to have relatively fast and smooth convergence behaviour, less computation time, as well as good accuracy, and thus the potential of solving real world problems. The performance of SDA particularly on the convergence speed and fitness accuracy is mostly determined by two important constant parameters of spiral model namely spiral radius, $r$ and angular displacement, $\theta$. Selection of suitable values for both constants is very crucial since defining small values for $r$, and $\theta$ may increase the convergence speed but it may also trap the agents to local optima and vice versa. These two parameters also cause all the agents to have constantly varying step size throughout the operation regardless of their distance from optimum location. In other words, the convergence rate for all agents towards the best location is uniform. This may also limit the movement of the agents within the search area hence may potentially lead them to get trapped into local optima. The problem may be solved by introducing an effective way of varying $r$ and $\theta$ or improving the exploration by adopting a strategy from other metaheuristic algorithms.

A study on performance comparison between the SDA and other well-known metaheuristic algorithms was conducted by Tamura and Yasuda, (2011b) and it was found that the SDA has better performance compared to PSO and showed competitive result compared to DE. In the literature, there are several techniques proposed by researchers to improve the performance of SDA. Some of these have successfully employed the improved SDA to solve real world problems. Nasir et al., (2012a) have proposed hybrid spiral-dynamics bacteria-chemotaxis (HSDBC) in which a chemotaxis scheme of bacteria is incorporated into SDA to enhance the exploration strategy. The bacteria motion is designed such that they tumble and swim in a spiral form with the same or different value of spiral radius or angular displacement. Nasir and Tokhi, (2013a) made a further study on the algorithm, replacing the spiral chemotaxis scheme to random tumble and swim approach. Moreover, to avoid oscillation in the convergence and to achieve optimum solution, the authors introduced the best fitness and the best optimum location terms in the chemotaxis phase. Nasir et al., (2012c) proposed adaptive versions of SDA where the spiral radius or angular displacement was made adaptive with fitness cost through linear and nonlinear mathematical formulations as well as fuzzy logic based relationships. Nasir et al., (2012b) further proposed a hybrid SDA approach namely hybrid spiral-dynamic bacteriaforaging (HSDBF), which synergised chemotaxis phase of bacteria through spiral tumble and swim with HSDBC algorithm to balance between the exploration and exploitation strategies. However, the algorithm was limited to low dimension problems only. The HSDBF implements a spiral approach in both tumble and swim for the exploration phase, followed by HSDBC for the exploitation phase. A deterministic approach is applied in both exploration and exploitation phases where in both phases the motion of bacteria is guided by the fittest bacteria in that particular iteration. More importantly, in the exploration phase of the HSDBF, it is hard to determine the bacteria motion if their next position is not a nutrient rich location and for higher dimensional problems, the algorithm may possibly fail to converge to a global optimum solution.

This paper presents a novel hybrid bacteria-chemotaxis spiral-dynamic (HBCSD) algorithm, where a BFA is synergised with SDA with the aim to improve the performance mainly on the accuracy and the convergence speed as well as to eliminate the drawbacks of its original constituent algorithms. The algorithm was initially reported briefly in a conference publication by the authors (Nasir et al., 2013b), and this paper provides a more comprehensive study of the algorithm with application to dynamic modelling of two types of flexible systems with different dynamic behaviours and complexity. The rest of the paper is organised as follows: The experimental rigs used in this work are described in Section 2. Section 3 describes the proposed hybrid algorithm. Section 4 presents application of the HBCSD to optimise parameters of high order ARX models, one for each of the highly nonlinear flexible systems. Results and discussion are presented in Section 5, and the main conclusions of the paper are drawn in Section 6.

\section{THE EXPERIMENTAL RIGS}

Two types of flexible systems that have different dynamic behaviours are considered in this work. The first system is a flexible robot manipulator and the second is a TRS, which replicates the behaviour of a helicopter in a laboratory setting. 


\subsection{FLEXIBLE MANIPULATOR SYSTEM}

A schematic diagram of the flexible manipulator is shown in Figure 1(a) (Azad, 1994). The system consists of a single flexible link made of aluminum beam and attached to an electromechanical motor. A U9M4AT type printed circuit board with bi-directional drive amplifier is used to rotate the motor shaft in both counterclockwise and clockwise directions. As there are three outputs of interest to be gauged from the system, three different sensor units are attached to the body of system. An accelerometer is placed at the tip (end-point) of the beam and used to measure end-point acceleration while an encoder with a resolution of 2,048 pulses/revolution and a tachometer are attached to motor shaft and used to measure hub-angle and hub-velocity respectively. Moreover, a personal computer (PC) embedded with Pentium Celeron $500 \mathrm{MHz}$ processor is connected with PCL818G interfacing unit to the flexible manipulator system. Matlab/Simulink software installed in the PC is used as a tool for controlling and manipulation of the system. The physical parameters of the beam such as length, $l$ width, $w$ and thickness, $h$ are $960 \mathrm{~mm}, 19.008 \mathrm{~mm}$ and $3.2004 \mathrm{~mm}$ respectively, the Young modulus, $E=71 \times 10^{9}$, the second moment of inertia, $I_{b}=0.04862 \mathrm{~kg}-\mathrm{m}^{2}$, mass-density $/$ volume, $\rho=2,710 \mathrm{~kg}-$ $\mathrm{m}^{-3}$ and hub inertia, $I_{h}=5.86 \times 10^{-4} \mathrm{~kg}-\mathrm{m}^{2}$.

\subsection{TWIN ROTOR SYSTEM}

The laboratory-scale TRS designed by Feedback Instrument Ltd. is used in this work (Feedback, 1996). A schematic diagram of TRS is shown in Figure 1(b) (Toha et al., 2012). It consists of the main rotor and tail rotor at both ends of horizontal beam pivoted on a base of the system. The main rotor blade rotates about yaw-axis while the tail rotor blade rotates about pitch-axis causing the system to move in vertical and horizontal directions respectively. The flexible motion due to unbalanced mass distribution on the system produces vibration while the system is in operation. Moreover, due to aerodynamic force, the structure suffers from deflection in both the vertical and horizontal directions. In the experimental work for the TRS, a random signal that is equivalent to electrical motor voltage is separately on the vertical and horizontal axes. The main rotor and tail rotor of the system are rotated about the vertical axis or yaw axis and the horizontal axis or pitch axis respectively. Thus, the main rotor drives the vertical movement of the system about pitch axis while the tail rotor drives the horizontal movement about yaw axis. In this information processing stage, the movement of the beam that holds the main and tail rotors about pitch and yaw axes is kept limitless. This is to ensure all dynamic behaviour of the system can be acquired at the maximum range. In this system, dynamic coupling effect of the vertical input to the horizontal output and coupling effect of the horizontal input to the vertical output must be taken into account. The literature shows that there is interaction between vertical input to the horizontal output but very small interaction between horizontal input to the vertical output and this is thus negligible (Ahmad et al., 2001).

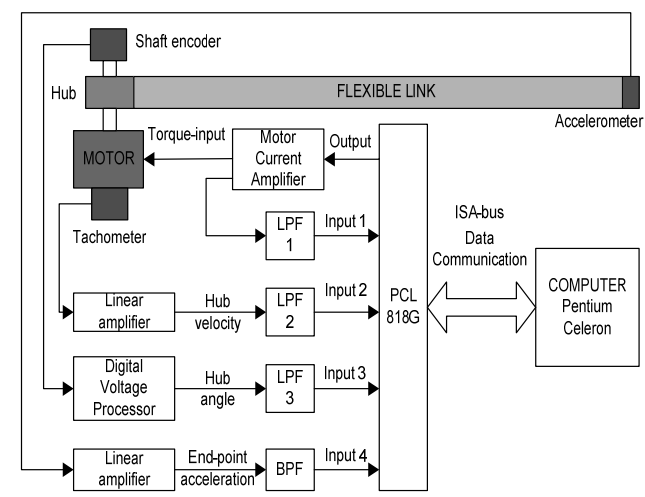

(a)

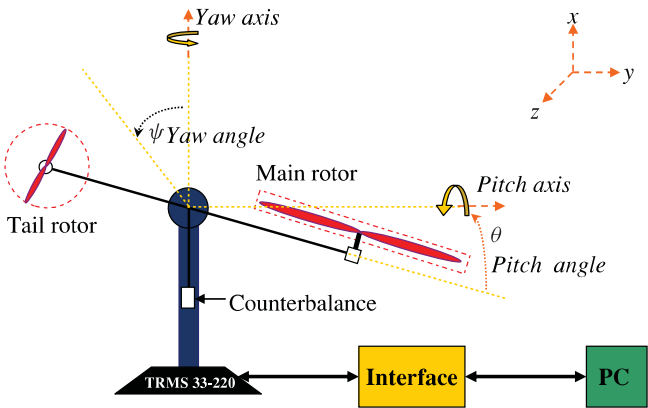

(b)

Fig. 1. (a) Schematic diagram of flexible manipulator, (b) Schematic diagram of TRS.

\section{THE PROPOSED HBCSD ALGORITHM}

In order to efficiently apply the BFA and SDA to solve real world problems, a novel strategy hybridising between the two algorithms is proposed in this section. In the proposed HBCSD algorithm, BFA and SDA are combined so as to balance the exploration and exploitation parts of the constituent algorithms. This algorithm is designed such that bacteria chemotaxis phase represents an exploration part while the SDA represents the exploitation part in the search operation.

Bacteria chemotaxis phase that consists of random tumble and swim actions is defined with large step size and must be placed in the first part of the algorithm. This is to ensure the bacteria can cover the search area thoroughly and also to expedite the algorithm convergence as bacteria tumble and 
swim with a large step. In this part, bacteria step size, $C$ and total number of chemotaxis $N_{C}$ are two important and crucial parameters that must be determined by the user. They must be defined such that the bacteria should have enough time to cover the whole search area and to reach the optimum point in the exploration stage. In other words, for a certain value of $C$, the $N_{C}$ is considered as the best if the exploration part is terminated when the convergence just reaches optimum value or starts to oscillate at the same location giving a similar fitness. On the other hand, the value of $C$ must not be too large so that to ensure the algorithm can converge to an optimum location in the exploration phase. The numerical value of both parameters may be different for different problems. At the end of exploration part, the local best location found by each bacterium is then stored and is used as the initial position of each bacterium in the second part of the algorithm. In the proposed HBCSD, the reproduction and elimination-dispersal phases of BFA are excluded as these will simplify the algorithm and reduce the total computation time of the algorithm. Nevertheless, they are replaced by another promising and effective approach that complements the exploration process performed through the chemotaxis strategy.

The second part of the HBCSD is exploitation part where at this stage, bacteria motion is done based on spiral model in SDA and guided by a global optimum point found in that particular iteration. The search operation of this part focuses on both global and local operations. This is because at the initial phase of spiral motion, the bacteria move diversely with a large step size towards a global optimum point while at the final phase of the spiral motion, bacteria move with a smaller step size to converge towards global optimum location. Moreover, the diversification is very important as the bacteria may find any better position along the way to the global optimum point in a wider space rather than simply move directly towards the goal point. It is worth noting that, at this point, all bacteria have explored the whole search area. Therefore, in HBCSD, the centre point of spiral model is considered as global optimum location after exhaustive search done by chemotactic strategy rather than simply picking up randomly from a search area. Since the step size is dynamically varied as the iteration progresses, the bacteria can easily reach any remote area or location that cannot be reached during exploration. It also may eliminate the oscillation problem that arises when a large value of $C$ is defined in the exploration part.

Moreover, to make the search operation more efficient, the spiral radius and angular displacement of the spiral model are adaptively defined based on linear relationship with respect to fitness of each bacterium. Incorporation of this approach into the spiral model can help to improve the algorithm performance in terms of convergence speed and fitness accuracy. The mathematical formulation for the linearly adaptive relationship is defined as:

$$
r_{l a}=\left(\left(r_{l}-r_{u}\right) /\left(1+\left(\frac{c_{1}}{c_{2}\left|f\left(x_{i}(k)\right)\right|}\right)\right)\right)+r_{u}
$$

where $r_{l a}$ is linear adaptive spiral radius, $\theta_{l a}$ is linear adaptive angular displacement, $c_{1}$ and $c_{2}$ are positive constants and $\left|f\left(x_{i}(k)\right)\right|$ is absolute fitness value of a particular point. $r_{u}$ and $r_{l}$ are maximum radius and minimum radius of spiral path trajectory for a particular point respectively.

It is important to note that, in the exploration part of HBCSD, the bacteria move independently of one another in a random manner and they freely determine their own direction. In the exploitation part, bacteria motion is guided toward a goal point in a spiral manner, which is a kind of deterministic approach. Combination of these two approaches may help the algorithm to avoid local optima and have faster convergence speed. The parameters used in n-dimensional HBCSD algorithm are described in Table 1 and the flowchart of the algorithm is shown in Figure 2.

Table 1. Parameters used in the HBCSD algorithm.

\begin{tabular}{|c|l|c|l|}
\hline Parameter & \multicolumn{1}{|c|}{ Description } & Parameter & \multicolumn{1}{|c|}{ Description } \\
\hline$f\left(x_{i}(k)\right)$ & $\begin{array}{l}\text { Fitness of } i^{\text {th }} \text { point in } k^{\text {th }} \\
\text { generation. }\end{array}$ & $r$ & $\begin{array}{l}\text { Spiral radius to be replaced by linear } \\
\text { adaptive. }\end{array}$ \\
\hline$s$ & Index of number of swim. & $\theta_{i, j}$ & $\begin{array}{l}\text { Search point angular displacement on } \\
x_{i}-x_{j} \text { plane around point of origin. }\end{array}$ \\
\hline$C$ & Bacteria step size. & $k_{\max }$ & Maximum number of iteration. \\
\hline$N_{C}$ & Number of chemotaxis. & $x^{*}$ & $\begin{array}{l}\text { Centre point of spiral model or global best } \\
\text { position. }\end{array}$ \\
\hline$N_{s}$ & Number of swim. & $x_{i}(k)$ & Position of $i^{\text {th }}$ point in $k^{\text {th }}$ generation. \\
\hline$i$ & $\begin{array}{l}\text { Index of number of search } \\
\text { point. }\end{array}$ & $I_{n}$ & Identity matrix with $n \times n$ dimensions \\
\hline
\end{tabular}




\begin{tabular}{|c|l|c|l|}
\hline Parameter & \multicolumn{1}{|c|}{ Description } & Parameter & \multicolumn{1}{c|}{ Description } \\
\hline$k$ & Index of number of iteration. & $P_{i}^{n}($ Best $)$ & $\begin{array}{l}\text { Optimum bacteria location found in } \\
\text { exploration phase. }\end{array}$ \\
\hline$m$ & Number of search point. & $R^{n}$ & $\begin{array}{l}\text { Composition of rotational } n \times n \text { matrix } \\
\text { based on combination of all } 2 \text { axes. }\end{array}$ \\
\hline
\end{tabular}

It is noted that the algorithm consists of exploration part through random tumble and swim action in the first phase and exploitation part, which is performed by SDA in the second phase. In the second phase, all the local best bacteria locations found in the exploration part are set to be the initial locations of bacteria in the exploitation part. Then, the best location among the optimum locations is set as the centre point of the spiral. Moreover, the adaptive spiral radius and angular displacement are applied in the second phase.

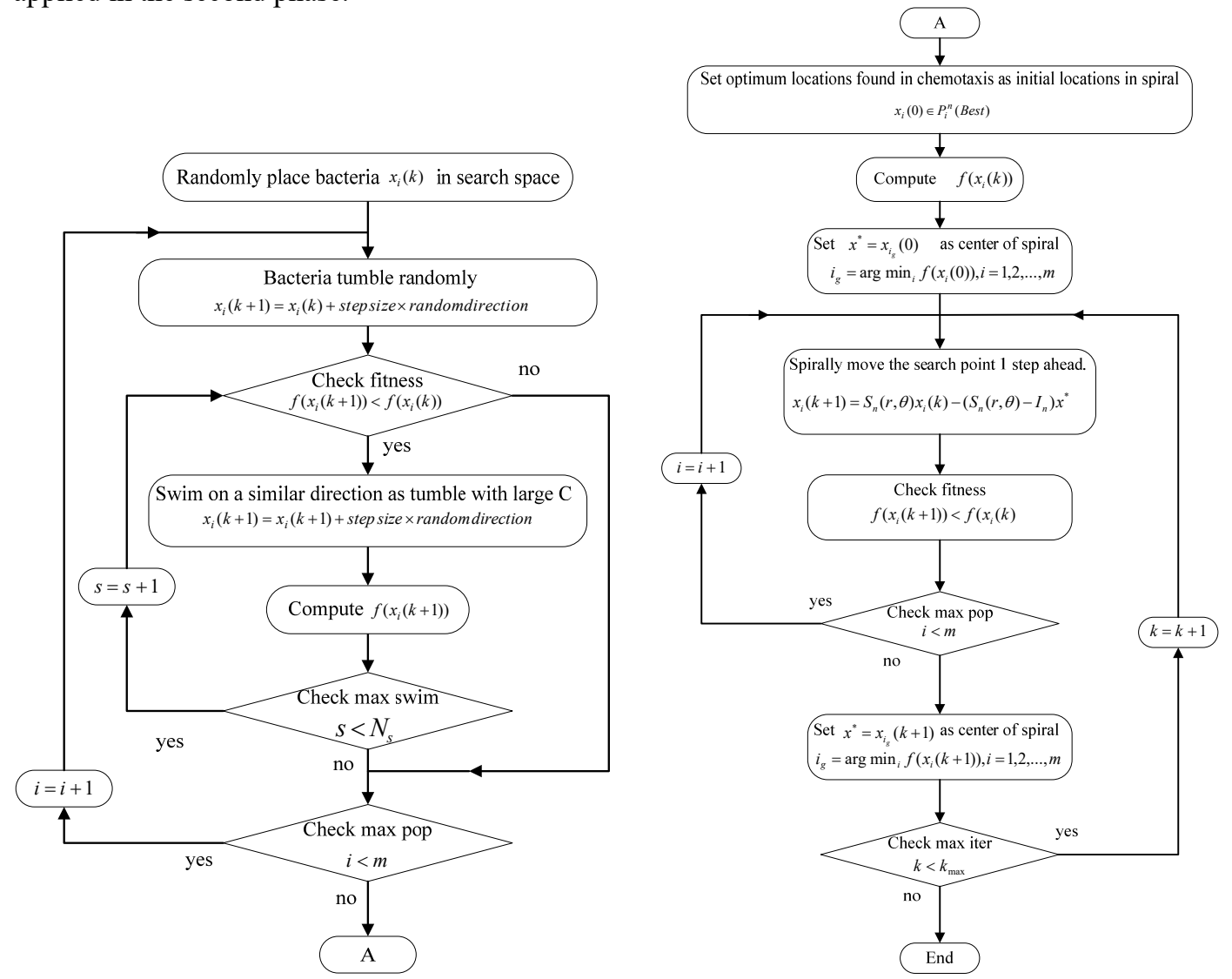

Fig. 2. Flowchart of hybrid bacteria-chemotaxis spiral-dynamic algorithm.

\section{MODEL CONSTRUCTION}

This section deals with data collected from actual hardware during experimentation for both the flexible manipulator system and the TRS. Then, modelling and validation of dynamic models for the two systems using the proposed HBCSD and its constituent algorithms, namely SDA and BFA, are discussed in detail.

\subsection{PRELIMINARY EXPERIMENTATION}

In the case of flexible manipulator, there are one input and three output responses to be observed. Here, a random signal input is applied to the system and this is represented as electrical motor torque. The amplitude of the torque was considered as $\pm 0.3 \mathrm{Nm}$. The measured output responses of the system comprise the hub-angle, hub-velocity and end-point acceleration. However, in this work, the end-point acceleration is chosen as the output response of interest since it dominantly represents the vibrational behaviour of the system. In real world applications, the capability of a controller in dealing with suppressing vibration at the end-point is of main concern. Therefore, the identification exercise was implemented to acquire dynamic model of the system from input torque to end-point acceleration output, here referred to as end-point acceleration model. A total of 2,300 experimental input-output data was collected to estimate the end-point acceleration model of the flexible manipulator. The first 1,500 data was used in the modelling phase while the remaining unused 800 data was used in the validation phase. 
For the TRS, the vibrational behaviour dominantly occurs at the pitch angle due to input in the vertical channel and therefore is chosen as the output of interest. A random signal \pm 1.0 radian with a sampling time, $T_{s}$ of 0.1 second or sampling frequency, $f_{s}$ of $10 \mathrm{~Hz}$ was used as input in the vertical channel of the system. A total of 8,000 actual input-output data was recorded to estimate the vertical channel model of the TRS. The first two-thirds of the data were used in the modelling phase while the remaining data was used to test the validity of the estimated model.

\subsection{MODEL STRUCTURE FORMULATION}

Formulation of the model structure is an essential step in a system identification procedure since it characterises the dynamic model of a system under study. Choosing the right structure and the best order are key to the performance for an estimated model and give true description about a system or process. A structure with low order that is too simple may lead to an inaccurate model, while a structure with high order that is too complex may lead to a very complex model. Both cases are not favoured in controller design since inaccurate model formulation clearly leads to poor control performance. On the contrary, determination of optimum parameters for a very complex model with high order is a very difficult task and requires more computing time. Moreover, high computing time might be needed when the model is used in a simulation software to design a controller. Therefore, compromises must be made in formulating the right model structure. The unknown parameters of the chosen model can be determined by representing them as coefficients in the numerator or denominator of a transfer function. If no prior information and physical description of a system is available, a black-box model approach can be used as an identification technique (Sjoberg et al. 1995). It is found in the literature that different types of model structure have been utilised to estimate dynamic model of flexible manipulator and TRS. The latest study by Supriyono and Tokhi, (2012) indicates that ARX model, which has a simple structure and relatively low computational cost can offer similar good performance in case of a flexible manipulator system. Similar conclusion has been drawn by Toha et al., (2012) with the use of ARX model for the TRS. Considering the performance and computational cost of the optimisation algorithm, in this work, the ARX model structure was determined the best candidate for both the flexible manipulator and the TRS. The general mathematical expression of the ARX model structure can be written as (Ljung, 1999):

$$
\widehat{y}(t)=-\sum_{i=1}^{N} a_{i} y(t-i)+\sum_{j=0}^{M} b_{j} u(t-j)+\eta(t)
$$

where $a_{i}$ and $b_{j}$ are the output and input coefficients, $N \geq M$ are the number of coefficients for the output and input samples, $\eta$ is the noise or disturbance and $\hat{y}, y$ and $u$ are the predicted output, measured output and measured input respectively. Assuming the actual model of the system is very good, then the measured output is highly dependent on the excited input and previous measured output and thus the noise term in the ARX expression can be neglected (Supriyono and Tokhi, 2012; Toha et al., 2012). In discrete transfer function form, the general expression of ARX structure is represented as:

$$
\frac{Y(z)}{U(z)}=\left(\sum_{j=0}^{M} b_{j} z^{-j}\right) /\left(1+\sum_{i=1}^{N} a_{i} z^{-i}\right)
$$

where $N$ is the order of the transfer function and the number of poles present in the system. An accurate and stable dynamic model of a system can be developed by determining the unknown coefficients of the denominator and numerator through an optimisation technique. The error of the optimisation algorithm for the modelling exercise can be regarded as the difference between the actual output and the predicted output, $e$ as:

$$
e(t)=y(t)-\widehat{y}(t)
$$

The error and accuracy have an inverse relationship where if the error value is reduced then the accuracy of the predicted model is improved. Moreover, by performing validation test, the accuracy of the estimated model can be evaluated. In practical terms, the selection of the model structure, order of transfer function and determination of parameters are done iteratively (Torsten and Petre, 2001). In this work, with reference to previous study on the same system, the order of the transfer function for end-point acceleration of flexible manipulator was found at its best performance when defined as eight. On the other hand, a fifth-order transfer function was found to be the best for the vertical channel dynamic model of the TRS. 


\subsection{HBCSD - BASED MODELLING}

The main concern in the modelling part is determination of the unknown parameters $a_{i}$ and $b_{j}$ in the ARX structure defined previously. The parametric modelling strategy with optimisation employed for both systems is depicted in Figure 3.

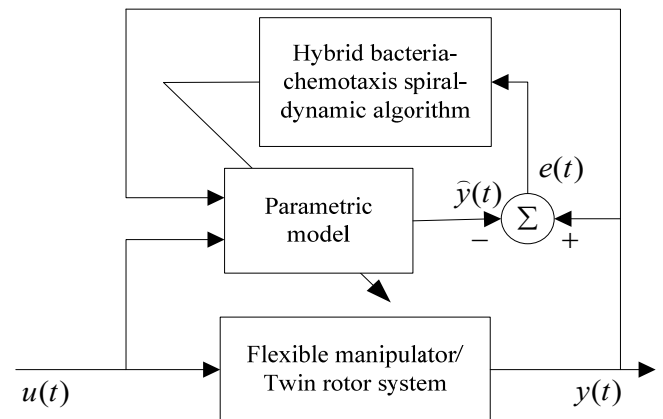

Fig. 3. Block diagram for parametric modelling with optimisation algorithm.

The $\hat{y}(t)$ in Figure 3 is the predicted output while $y(t)$ and $u(t)$ are recorded output-input from the experimental system. The $e(t)$ is the modelling error and is used in formulating the cost function for the optimisation algorithm. There are different types of error functions that can be employed in the optimisation algorithm. In this work, root mean square error with gain function was selected as a minimisation cost function. In dealing with constraints in the modelling phase, a penalty function can be incorporated into the cost function. For a stable discrete transfer function of a dynamic system, all poles must lie within the unit circle (in the z-plane). If there is any pole of the discrete transfer function outside the unit circle, then the solution must be disregarded even if the magnitude of the error function is very small. The solution is penalised by adding a large constant value to the error function. The cost function with penalty approach is thus defined as:

$$
\begin{gathered}
f(t)=\left(\frac{1}{K} \sqrt{\sum_{t=1}^{K} e^{2}(t)}\right) w \quad \text { if all poles }<1 \\
f(t)=\left(\frac{1}{K} \sqrt{\sum_{t=1}^{K} e^{2}(t)}\right) w+\text { penaltygain else }
\end{gathered}
$$

where $K$ is total number of sampled data, $e$ is the difference between actual and predicted outputs as in Eqn. (4), $w$ is a gain factor and poles are roots of the denominator polynomial in Eqn. (3). Since the error is squared, this cost function has more chances to reduce large errors. The gain factor is used to amplify the function value in case extremely small error is produced. The penalty gain in Eqn. (6) can be defined as a large constant value. In this work, the penalty gain and $w$ have been defined as $1 \times 10^{9}$ and $1 \times 10^{3}$ respectively. Incorporating such approach into the optimisation algorithm may lead to extra challenge but a stable solution can always be preserved throughout the whole set of iterations.

\subsection{HBCSD - BASED VALIDATION}

In general, three types of tests are conducted to evaluate the adequacy of a predicted model to represent the actual system. Only if the estimated model passes all the tests, then it can be considered as adequate to represent the actual system. However, if it does not pass at least one of the tests, then the modelling exercise must be repeated.

(a) Experimental input-output data. The actual input-output data recorded during experimental exercise can be separated into modelling and validation parts. In practice, the first two-thirds of the data are used to estimate the model in the modelling phase while the remaining data can be used to check the predicted model validity in the validation phase (Ljung, 1999). Ideally, the output of the predicted model must portray exactly the same signal as the second portion of the actual output if the actual input is applied to the estimated model. A block diagram for linear parametric system identification used to validate the estimated model of a system is depicted in Figure 4. 


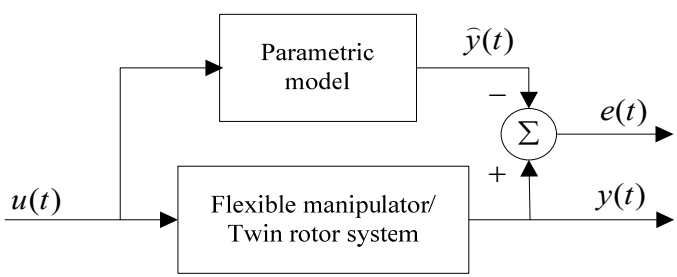

Fig. 4. Block diagram to validate the estimated model in linear parametric system identification.

(b) Stability test. The stability test is normally conducted through determination of poles of a discrete or a continuous transfer function. A discrete model is considered as stable if all poles lie within the unit circle in the z-plane. On the other hand, a continuous model is regarded as stable if all poles lie in the left hand s-plane.

(c) Correlation test. The correlation test is a popular approach used to test the adequacy or quality of the predicted model. It is regarded as model validity test to identify whether there are any un-modelled linear or non-linear terms in the residual, which normally lead to biased estimates. A linear timeinvariant model is considered valid if the following conditions are satisfied (Billings and Voon, 1986):

$$
\begin{array}{r}
\varphi_{\varepsilon \varepsilon}(t)=E[\varepsilon(t-\tau) \varepsilon(t)]=\delta(\tau) \\
\varphi_{u \varepsilon}(\tau)=E[u(t-\tau) \varepsilon(t)]=0, \forall \tau
\end{array}
$$

where $\varphi_{\varepsilon \varepsilon}(\tau)$ is the auto-correlation function of the error or residual, $\varepsilon(t), \delta(\tau)$ is impulse function and $\varphi_{u \varepsilon}(\tau)$ is cross-correlation function between input, $u(t)$ and residual, $\varepsilon(t)$. If the residuals are unpredictable from all linear and non-linear combinations of past inputs-outputs, the estimated model is considered as a valid model (Toha et al., 2012). For sampled input and output signals, the correlation function can be normalised within a range of \pm 1 . Moreover, the correlation between the variables can never be exactly zero for most lags and therefore, in practice, the $95 \%$ confidence interval is used as a basis to show the estimated correlation is significant. An estimated model is said to be a good model if the correlation plot does not significantly cross the $95 \%$ confidence boundary.

\section{RESULTS AND DISCUSSION}

This section presents results and associated discussion of dynamic modelling of the flexible systems. Comparative assessment of the optimisation algorithms under study is presented on the basis of accuracy or the ability of the algorithm to achieve the smallest error and the convergence speed to reach an optimum point. Moreover, the response of the estimated model in both modelling and validation phases is compared to the actual experimental data to assess how good the acquired model compared to the actual system is. The pole-zero map, correlation plot and frequency-domain plot are also shown to assess the stability, the adequacy and dynamic behaviour of the acquired model.

\subsection{DYNAMIC MODEL IDENTIFICATION FOR THE FLEXIBLE MANIPULATOR SYSTEM}

This section describes dynamic modelling for end-point acceleration of the flexible manipulator. In this work, the initial search points were placed randomly in a high dimension space and they were kept the same for all algorithms as the placement of initial positions affect the performance of the optimisation. This provides similar difficulty and equal chances to all algorithms to find the global optimum point.

\subsubsection{MODELLING PHASE FOR END-POINT ACCELERATION}

Naturally, vibration will occur when an external force is applied on a flexible structure. In the flexible manipulator, vibration occurs due to fast motion. This is measured by an accelerometer placed at the end point of the flexible beam. Previous studies have shown that the flexible manipulator system response consists of three main dominant modes within 0-100 Hz (Alam and Tokhi, 2007a). Thus, to model the end-point acceleration over $0-100 \mathrm{~Hz}$, at least three pairs of complex conjugate poles are required resulting in a sixth-order transfer function. However, incorporating the rigid body dynamics of the flexible manipulator, an eight-order ARX model is considered for end-point acceleration (Supriyono and Tokhi, 2012). Substituting $N=8$ and $M=7$ in Eqn. (3), the ARX model for the system can be represented in discrete transfer function form as:

$$
\frac{Y(z)}{U(z)}=\frac{b_{1} z^{7}+b_{2} z^{6}+b_{3} z^{5}+b_{4} z^{4}+b_{5} z^{3}+b_{6} z^{2}+b_{7} z+b_{8}}{z^{8}+a_{1} z^{7}+a_{2} z^{6}+a_{3} z^{5}+a_{4} z^{4}+a_{5} z^{3}+a_{6} z^{2}+a_{7} z^{1}+a_{8}}
$$


Therefore, there are 16 unknown parameters to be identified using the optimisation algorithms. The number of search points for SDA and number of bacteria for BFA and HBCSD were defined as 50 while 1,000 iterations were considered for SDA and HBCSD. The optimisation parameters for BFA were defined as $N_{s w}=4, N_{c}=30, N_{r e}=6$ and $N_{e d}=6$ hence giving 1,080 iterations in total for BFA, which is almost similar to the total iterations for SDA and HBCSD. This is also to ensure the bacteria have sufficient step size to complete the whole search operation. Moreover, this parameter setup enables the assessment and comparison of the algorithms performance in terms of convergence speed and fitness accuracy within 1,000 iterations can be conducted. Considering both speed and accuracy of BFA, various step sizes of the bacteria were iteratively tested and it has shown the best performance with $C=0.01$. On the other hand, after applying various radii and angles through exhaustive test, SDA has shown the best performance with $r=0.99$ and $\theta=0.381$ while for HBCSD, it has shown the best performance when the parameters were defined as $N_{s w}=4, C=0.01$, $N_{c}=500, \theta=0.34$ and $k_{\max }=500$. For the adaptive spiral radius of the HBCSD, the $r_{l}, r_{u}, c_{1}$ and $c_{2}$ were defined as $0.99,1,0.1$ and 0.3 respectively. The step size, $C$ and number of chemotaxis, $N_{c}$ in HBCSD were selected as 0.01 and 500 respectively to ensure it had at least similar convergence speed compared to BFA during the exploration process and at the same time able to achieve positions with good accuracy prior to the exploitation phase. Then, the rotational angle, $\theta$ must be selected such that it can perform a good exploitation process and able to achieve any difficult position in remote area as fast as possible. Other parameters in HBCSD were found by trial and error after performing exhaustive tests.

The convergence plots, time-domain and frequency-domain results for end-point acceleration of the flexible manipulator in the modelling phase thus obtained are depicted in Figure 5. Notice that at the initial phase, the SDA converged sharply towards better fitness location, and was faster than the other two algorithms. However, at iteration 700, HBCSD intercepted the SDA graph and it continuously searched the feasible region and converged to a better fitness location. On the contrary, at this point, the BFA and SDA gradually settled and trapped at local optima. The numerical results for the convergence plots are presented in Table 2. Here, the best fitness value for the whole computation and the speed of convergence at the iteration when the convergence plot intercepts the fitness value 35 are recorded. The best fitness value $f_{x}=22.99$ was achieved by HBCSD, followed by SDA and BFA with fitness values $f_{x}=32.41$ and $f_{x}=35.49$ respectively. The time-domain plots show that the response with HBCSD followed the actual response better than those with SDA and BFA.

Table 2. Numerical results of SDA, BFA and HBCSD for end-point acceleration.

\begin{tabular}{|c|c|c|c|}
\hline Algorithm & $\begin{array}{c}\text { Best fitness, } \\
f_{x}\end{array}$ & $\begin{array}{c}\text { Convergence steps } \\
\text { at 35 (Iteration) }\end{array}$ & $\begin{array}{c}\text { Range of modelling } \\
\text { error }\end{array}$ \\
\hline BFA & 35.49 & 1057 & {$[-0.1054,-0.0936]$} \\
\hline SDA & 32.41 & $\mathbf{6 4 5}$ & {$[-0.1100,0.0970]$} \\
\hline HSDBC & $\mathbf{2 2 . 9 9}$ & 698 & {$[-\mathbf{0 . 0 8 7 5 , 0 . 0 7 7 5}]$} \\
\hline
\end{tabular}

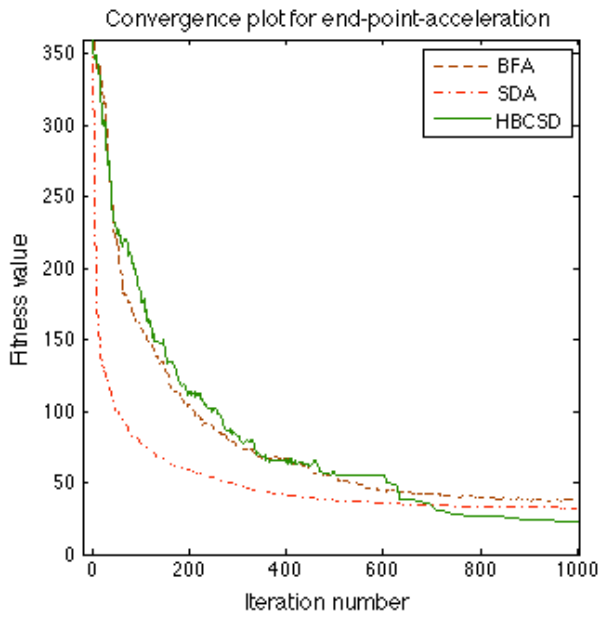

(a) Convergence plot

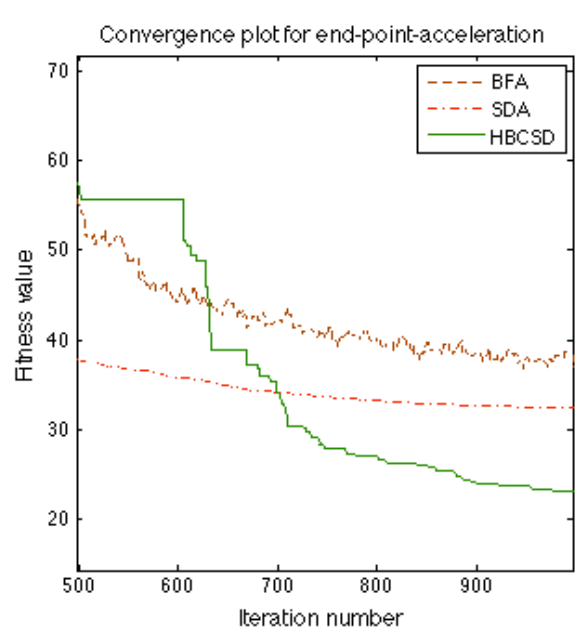

(b) Convergence plot (zoomed-in) 


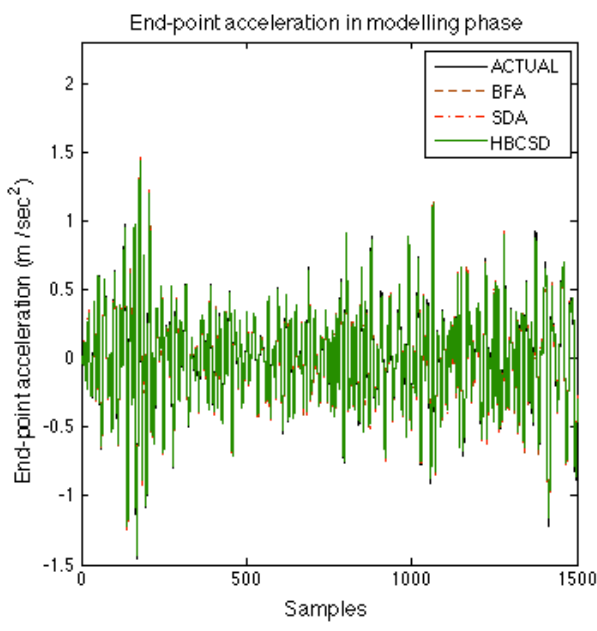

(c) Time-domain response

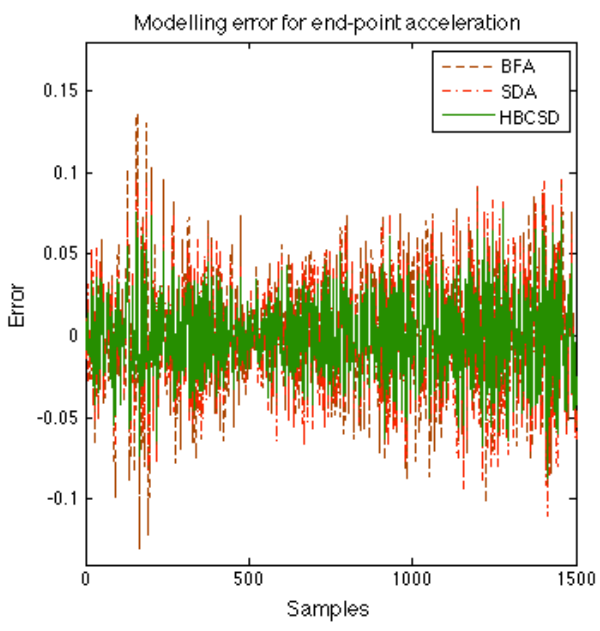

(d) Error

Fig. 5. Simulation results for end-point acceleration of flexible manipulator in the modelling phase.

Substituting the optimised parameters into the ARX model structure, the eight-order discrete transfer functions for the predicted end-point acceleration model optimised with a sampling period of 0.001 seconds (sampling frequency of $1,000 \mathrm{~Hz}$ ) are obtained as:

$$
\begin{aligned}
& H(z)_{B F A}=\frac{-0.02719 Z^{7}-0.0008001 Z^{6}-0.02813 Z^{5}-0.005624 Z^{4}+0.04217 Z^{3}-0.02064 Z^{2}+0.004525 Z+0.02602}{Z^{8}-1.748 Z^{7}-0.54 Z^{6}+0.5364 Z^{5}+0.08793 Z^{4}-0.3422 Z^{3}-0.01754 Z^{2}-0.1027 Z+0.2114} \\
& H(z)_{S D A}=\frac{0.01916 Z^{7}-0.01191 Z^{6}-0.005592 Z^{5}-0.01978 Z^{4}+0.003867 Z^{3}+0.01559 Z^{2}+0.006081 Z+0.01818}{Z^{8}-1.617 Z^{7}-0.3437 Z^{6}+1.848 Z^{5}-0.198 Z^{4}-0.9938 Z^{3}+0.103 Z^{2}+0.413 Z-0.102} \\
& H(z)_{H B C S D}=\frac{-0.00814 Z^{7}-0.003931 Z^{6}+0.0006345 Z^{5}+0.001083 Z^{4}-0.006531 Z^{3}+0.0009403 Z^{2}+0.01424 Z+0.01195}{Z^{8}-2.137 Z^{7}+0.9841 Z^{6}+0.9026 Z^{5}-0.5224 Z^{4}-0.3894 Z^{3}-0.06197 Z^{2}+0.5 Z-0.204}
\end{aligned}
$$

The pole-zero locations for the predicted discrete transfer functions of end-point acceleration models thus obtained are depicted in Figure 6. The marks ' $x$ ' and ' 0 ' represent pole and zero for the estimated model respectively while the dotted-line circle in the diagram represents a unit circle. It is noted that all the poles for each model were within a unit-circle indicating that they were all stable. Some of the zeroes were outside the unit-circle, which imply the presence of non-minimum phase behaviour.

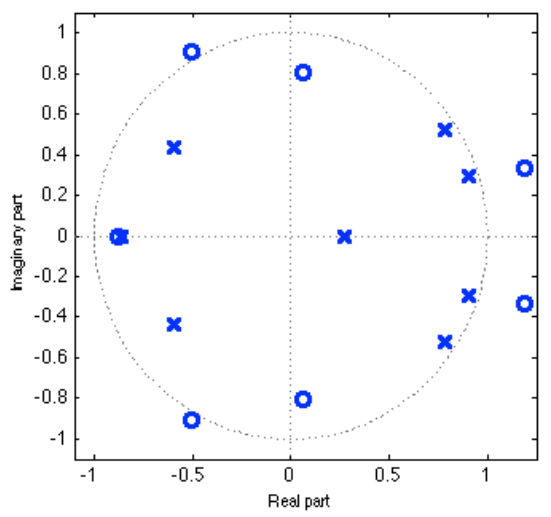

(a) SDA

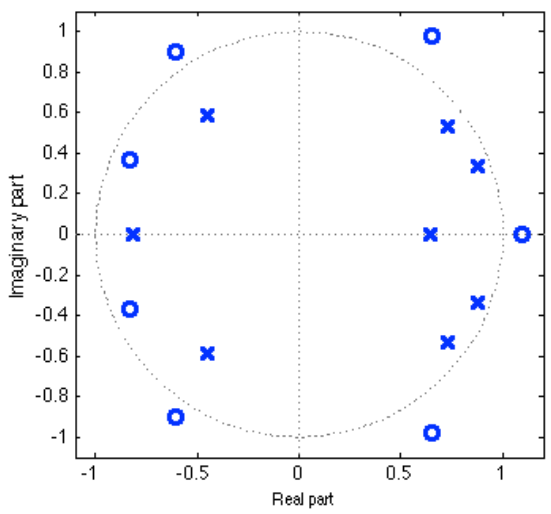

(b) HBCSD 


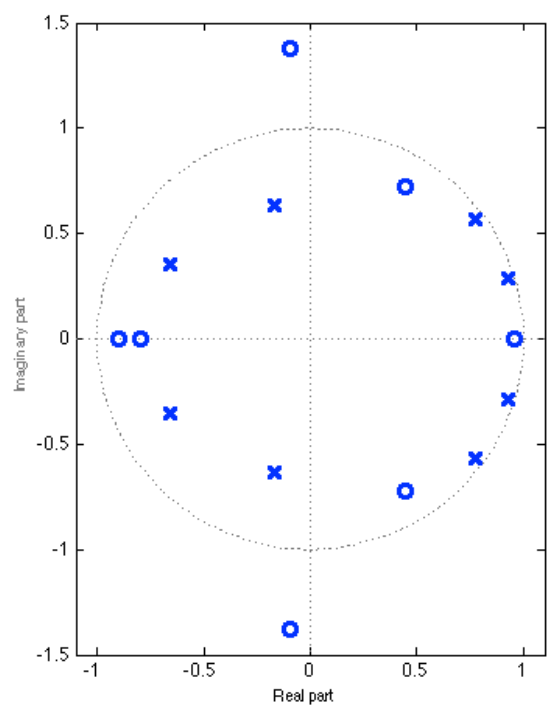

(c) BFA

Fig. 6. Poles and zeros location for the end-point acceleration model.

\subsubsection{VALIDATION PHASE FOR END-POINT ACCELERATION}

In the validation phase, the remaining unused experimental input-output data was used to test the closeness of the estimated model response to the actual system response. Then, all the data from this test were used to assess the auto-correlation and cross-correlation relationships of the response from estimated model. Results and graphs for the validation test are shown in Figure 7. In terms of timedomain response, it is noted that all the estimated model responses were approximately coinciding the end-point acceleration response from actual system and the error signal shows that HBCSD achieved the smallest error range indicating the best accuracy. Moreover, from the time-domain response (zoomed-in) graph, it is clearly evident that the end-point acceleration response using HBCSD algorithm was able to closely follow the actual response better than other responses. Their corresponding power spectral density (PSD) plots show three resonance modes at $11.72 \mathrm{~Hz}, 39.06 \mathrm{~Hz}$ and $66.41 \mathrm{~Hz}$. It is noted that the PSD of model response using HBCSD algorithm tracked the actual PSD better than the others.

The auto-correlation plots for the estimated models using BFA, SDA and HBCSD represent the relationship between end-point acceleration residuals, and are presented in Figure 8. Similarly, the cross-correlation plots represent relationship between end-point acceleration residual and the motor torque input, and are presented in Figure 8 . The dotted line in the figures represent $95 \%$ confidence boundary. It is noted that the auto-correlation and cross-correlation plots for all the estimated models were within the $95 \%$ interval, showing that the predicted models optimised by the optimisation algorithms were acceptable.

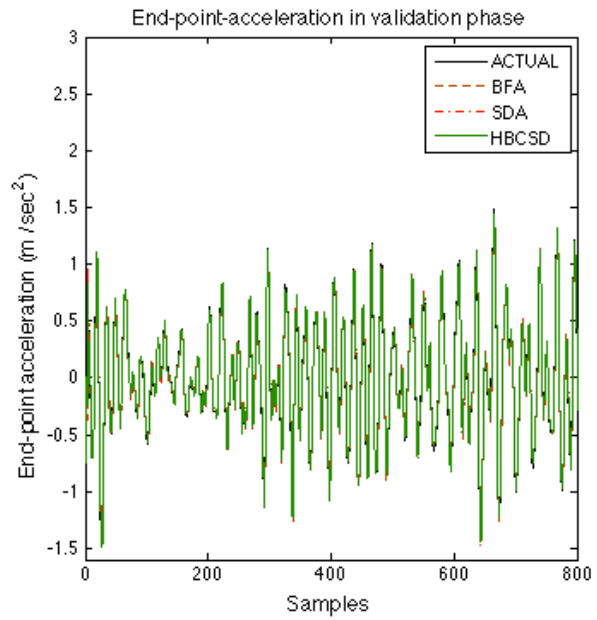

(a) Time-domain response

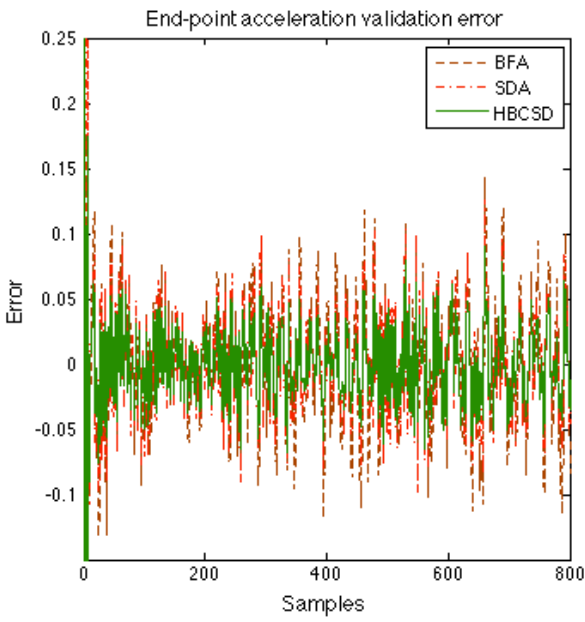

(b) Error 


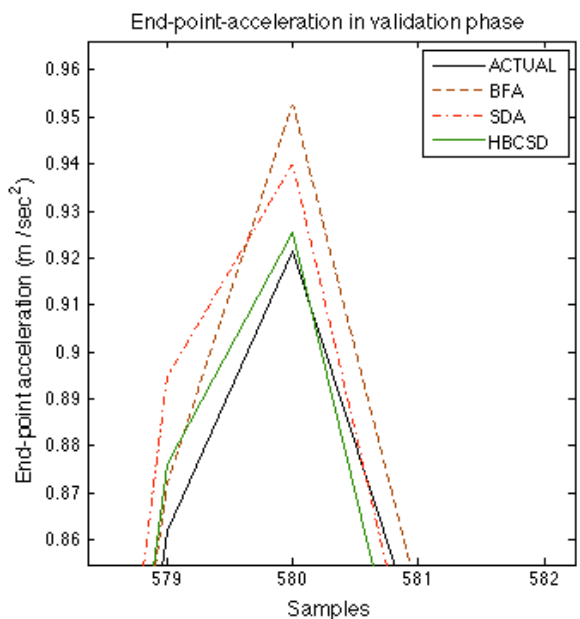

(c) Time-domain response (zoomed-in)

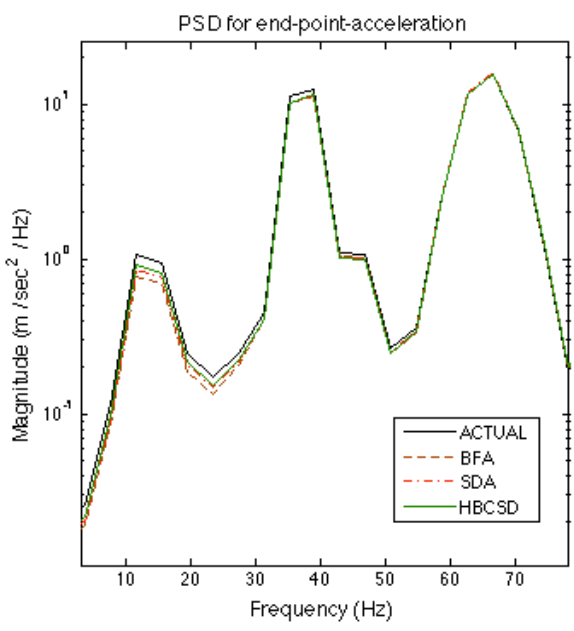

(d) Power spectral density

Fig. 7. Simulation results for end-point acceleration of flexible manipulator in the validation phase.
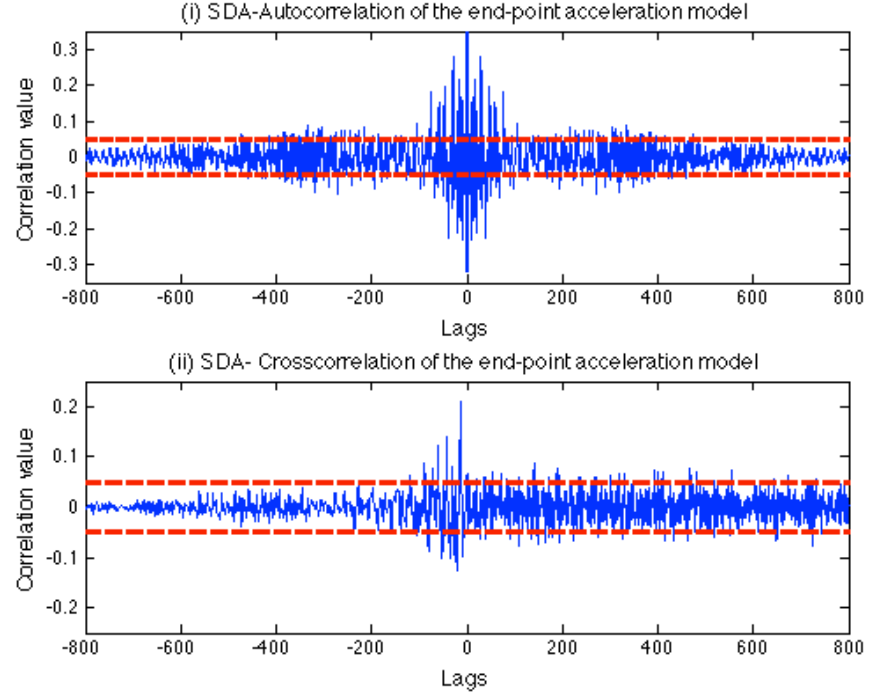

(a) SDA
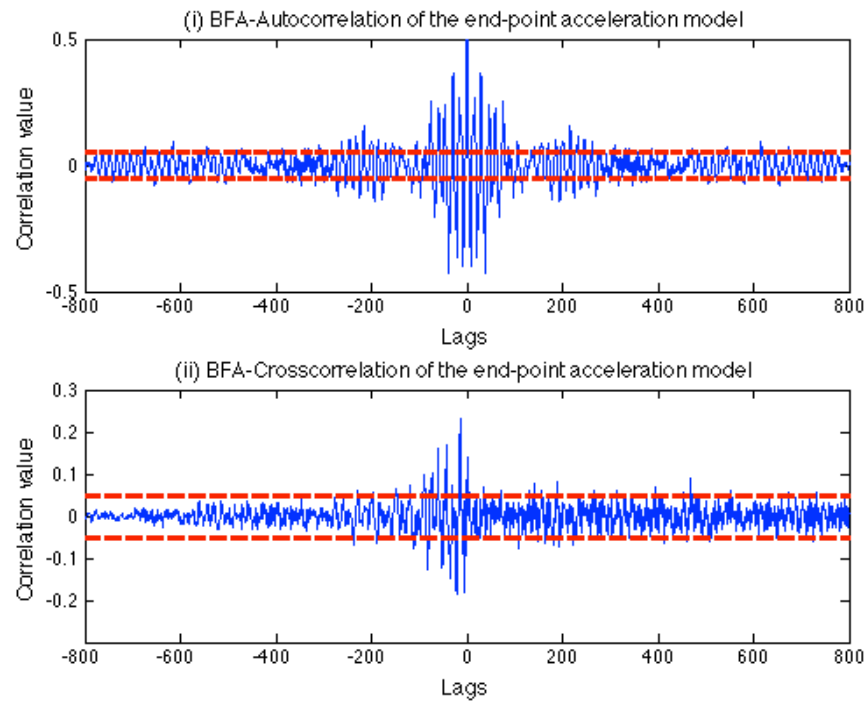

(b) BFA 


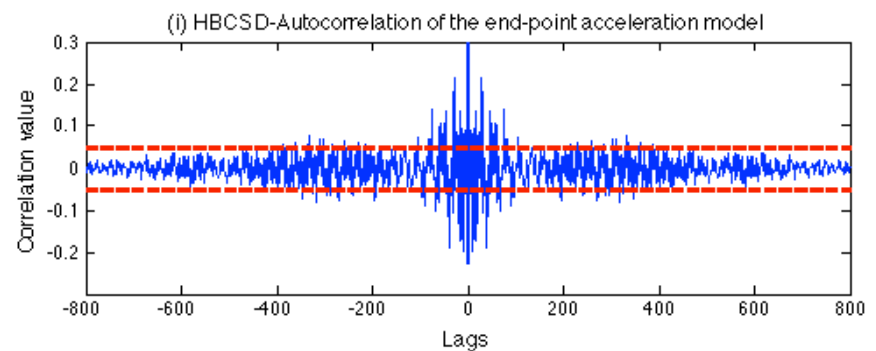

(ii) HBCSD-Crosscorrelation of the end-point acceleration model

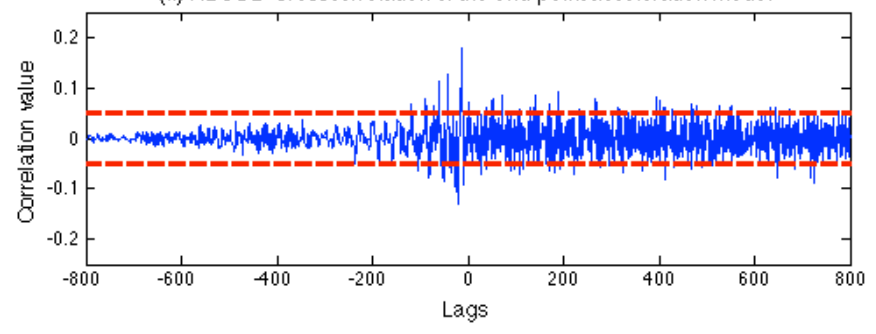

(c) HBCSD

Fig. 8. Autocorrelation and crosscorrelation for the end-point acceleration model of the flexible manipulator.

\subsection{DYNAMIC MODEL IDENTIFICATION FOR THE TWIN ROTOR SYSTEM}

This section presents dynamic modelling of vertical channel of the TRS. In this work, fifth-order ARX models are considered. Replacing the values of $N$ and $M$ in Eqn. (3) as 5 and 4 respectively, in discrete transfer function form, the ARX model for the system is represented as:

$$
\frac{Y(z)}{U(z)}=\frac{b_{1} z^{4}+b_{2} z^{3}+b_{3} z^{2}+b_{4} z+b_{5}}{z^{5}+a_{1} z^{4}+a_{2} z^{3}+a_{3} z^{2}+a_{4} z^{1}+a_{5}}
$$

Therefore, there are a total of 10 unknown parameters to optimise using the optimisation algorithms for vertical channel of TRS.

\subsubsection{MODELLING PHASE FOR VERTICAL CHANNEL}

The number of search points in SDA and HBCSD is equivalent to the number of bacteria in BFA and they were defined as 10 . They were defined the same to ensure a fair comparison can be conducted on the performance assessment. The number of iterations for SDA and HBCSD were kept the same as 200 while for BFA, it was 480 since it depends on $N_{c}, N_{r e}$ and $N_{e d}$. They were defined as $N_{s w}=4$, $N_{c}=30, N_{r e}=4$ and $N_{e d}=4$ to ensure the bacteria have enough iteration to complete the whole search operation hence achieve optimum performance. The selection of bacteria step size for BFA has been iteratively tested and it has the best performance when the step size was defined as $C=0.0175$. After performing exhaustive test, the parameters $r=0.98$ and $\theta=0.72$ were defined for SDA as they gave the best performance. The HBCSD had the best performance when the parameters were defined as $N_{s w}=4, C=0.04, N_{c}=50$ and $k_{\max }=150$. For the adaptive spiral radius of the HBCSD, the $r_{l}, r_{u}, c_{1}$ and $c_{2}$ were defined as $0.65,0.99,996$ and 0.97 respectively while $\theta_{l}=1.03, \theta_{u}=1.8, c_{1}=110$ and $c_{2}=0.98$ were defined for the adaptive angular displacement or rotational angle. The step size, $C$ for HBCSD was selected as 0.04 to expedite the algorithm convergence during the exploration phase. Consequently, the parameters for the HBCSD in the exploitation phase must be selected such that the bacteria can reach difficult position in remote area to complement the strategy in the exploration phase.

The numerical results for the convergence plot are shown in Table 3. Here, the best fitness value for the whole computation and the speed of convergence at a time when the convergence plot intercepts the fitness value 15 were recorded. Notice that the best fitness among those three algorithms was achieved by HBCSD with $f_{x}=8.80$. This was followed by SDA and BFA, with best fitness values of 12.69 and 14.32 respectively. Moreover, HBCSD acquired the fastest convergence speed at 83 iterations. The convergence plots for vertical channel, the predicted-actual plot for vertical channel output in the time-domain and their corresponding errors are depicted in Figure 9. It is clearly evident from the results that all the predicted outputs successfully followed the actual output of the vertical channel with very small error. The results show that HBCSD achieved the smallest error range and the 
corresponding model response successfully followed the actual response more accurately compared to others.

Table 3. Numerical results of SDA, BFA and HBCSD for vertical channel of TRS.

\begin{tabular}{|c|c|c|c|}
\hline Algorithm & $\begin{array}{c}\text { Best fitness, } \\
f_{x}\end{array}$ & $\begin{array}{c}\text { Convergence steps at } \\
f_{x}=\mathbf{1 5} \text { (Iteration) }\end{array}$ & $\begin{array}{c}\text { Range of modelling } \\
\text { error }\end{array}$ \\
\hline BFA & 14.32 & 407 & {$[-0.0729,0.0601]$} \\
\hline SDA & 12.69 & 149 & {$[-0.0413,0.0481]$} \\
\hline HSDBC & $\mathbf{8 . 8 0}$ & $\mathbf{8 3}$ & {$[-\mathbf{0 . 0 2 6 0 , 0 . 0 3 5 1 ]}$} \\
\hline
\end{tabular}

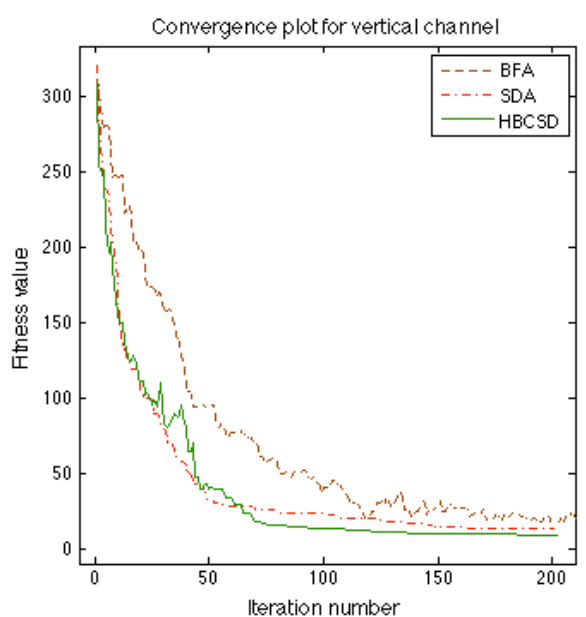

(a) Convergence plot

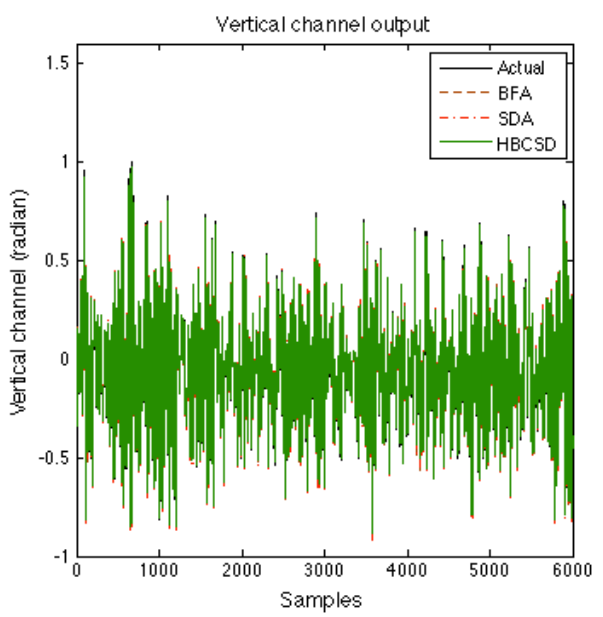

(c) Time-domain response

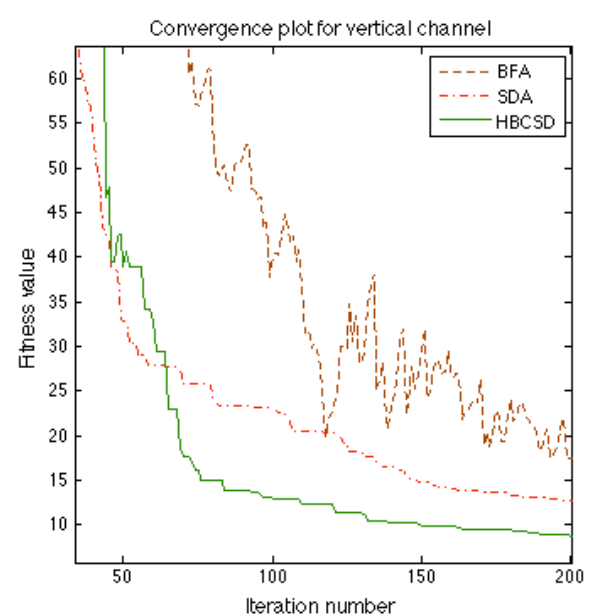

(b) Convergence plot (zoomed-in)

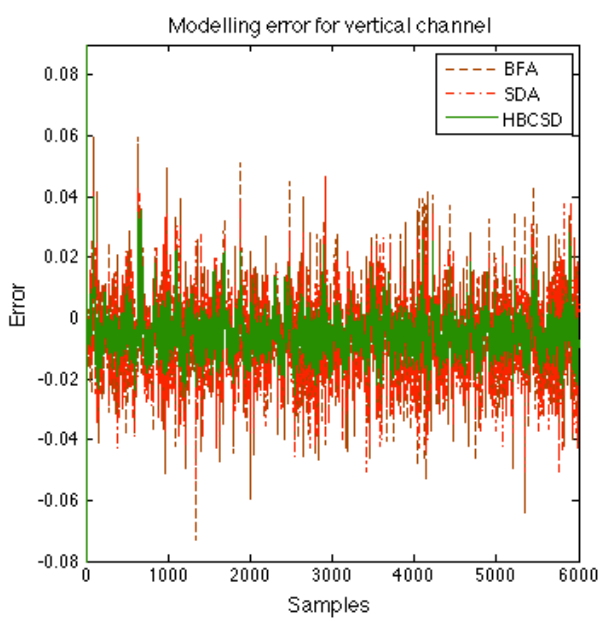

(d) Error

Fig. 9. Simulation results for vertical channel of TRS in the modelling phase.

The fifth-order discrete transfer functions for the predicted TRS vertical channel model optimised with sampling period of 0.1 seconds (sampling frequency of $10 \mathrm{~Hz}$ ) are obtained as:

$$
\begin{gathered}
H(z)_{B F A}=\frac{0.05855 Z^{4}+0.02523 Z^{3}-0.1124 Z^{2}-0.005456 Z+0.06812}{Z^{5}-1.224 Z^{4}+0.1875 Z^{3}-0.3414 Z^{2}+0.3839 Z+0.09502} \\
H(z)_{S D A}=\frac{-0.02553 Z^{4}+0.03134 Z^{3}-0.03532 Z^{2}+0.04564 Z+0.02174}{Z^{5}-0.8867 Z^{4}-0.6821 Z^{3}+0.6109 Z^{2}-0.239 Z+0.3122} \\
H(z)_{H B C S D}=\frac{0.01111 Z^{4}-0.03272 Z^{3}+0.01425 Z^{2}+0.007029 Z+0.02739}{Z^{5}-1.367 Z^{4}-0.04578 Z^{3}+0.4877 Z^{2}-0.09271 Z+0.0887}
\end{gathered}
$$

The pole-zero location for the estimated model is shown in Figure 10. The marks ' $x$ ' and ' $o$ ' represent pole and zero for the estimated model respectively while the dotted-line circle in the diagram 
represents a unit-circle. The pole-zero location shows that all the poles were within the unit-circle which indicates that the acquired models were stable. Some of the zeroes were outside the unit-circle, which imply non-minimum phase model behaviour.

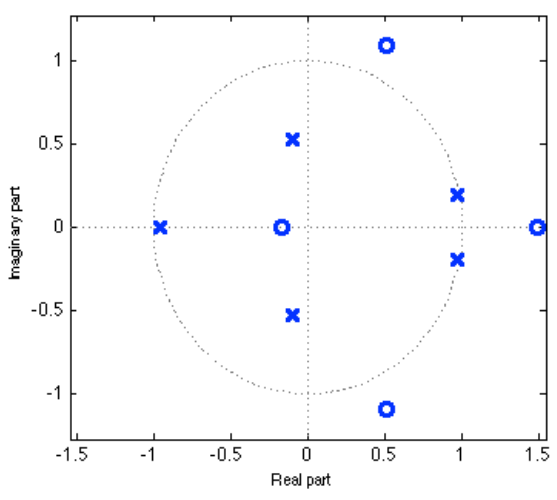

(a) SDA

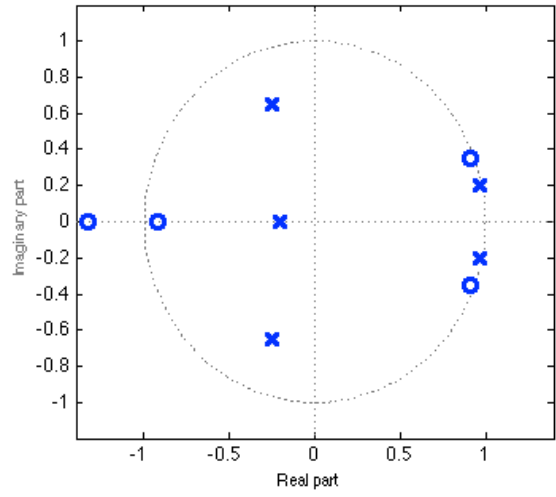

(b) BFA

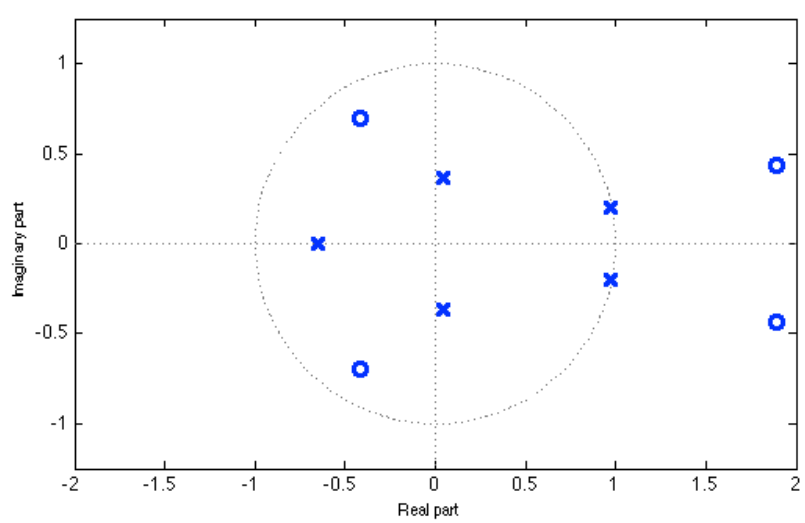

(c) HBCSD

Fig. 10. Poles and zeros location for the vertical channel model of TRS.

\subsubsection{VALIDATION PHASE FOR VERTICAL CHANNEL}

In the validation phase, the 2,000 unused experimental data was utilised to examine the closeness of the estimated model response to the actual response. The data and results produced by the test consequently were used to plot auto-correlation and cross-correlation of predicted model responses. Graphical results for vertical channel of TRS in the validation phase are depicted in Figure 11. Notice that, all the graphs almost overlapped with each other for the whole 2,000 samples and the error in the validation phase was very small within a range of $[-0.08,0.06]$. It is found that HBCSD had the smallest error range within [-0.059,0.042] while the largest error range within $[-0.070,0.053]$ was produced by BFA. It is clearly evident from the time-domain (zoomed-in) response that the vertical channel response produced by estimated model using HBCSD followed the actual response better than the other two responses. Their corresponding PSD plots showed one dominant resonance mode at $0.35 \mathrm{~Hz}$. 


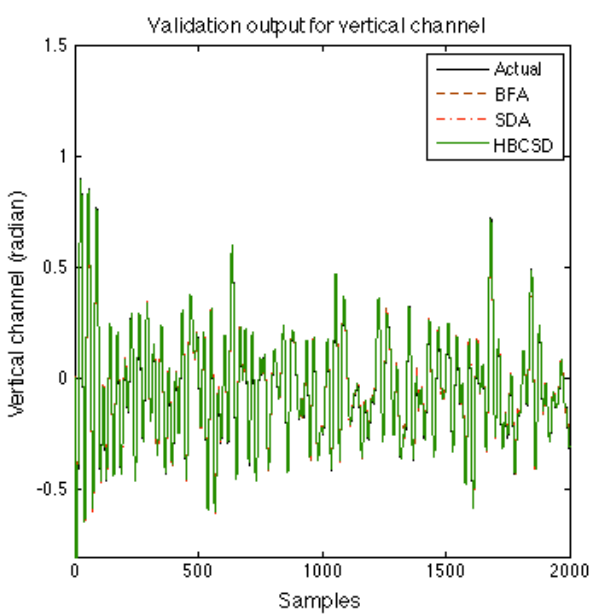

(a) Time-domain response

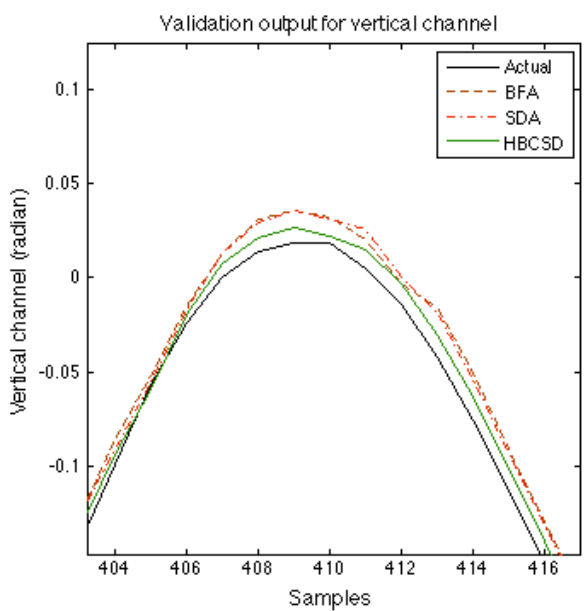

(c) Time-domain response (zoomed-in)

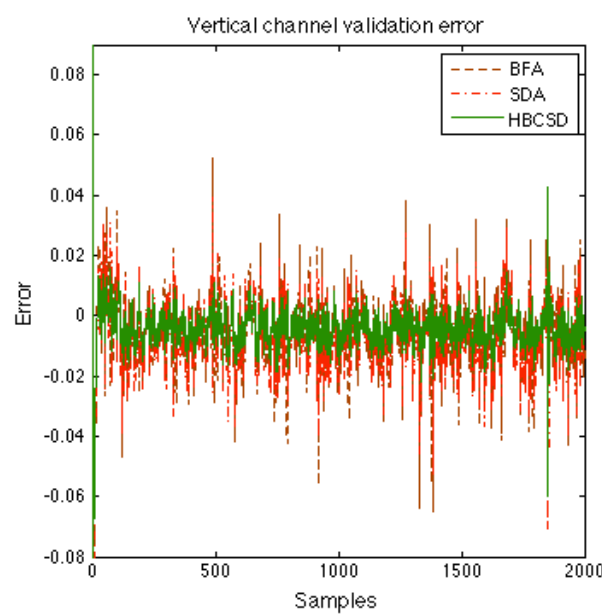

(b) Error

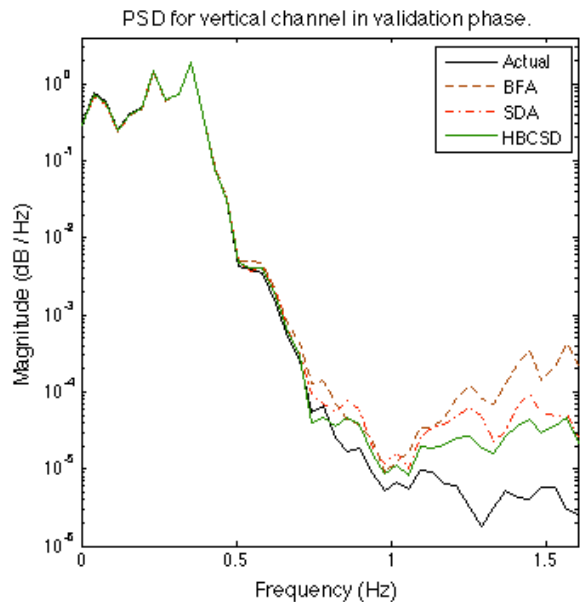

(d) Power spectral density

Fig. 11. Simulation results for vertical channel of TRS in the validation phase.

The auto-correlation plots for the estimated model using BFA, SDA and HBCSD represent those of the modelling error, and are presented in Figure 12. Similarly, the cross-correlation plots represent the relationship between the input and the modelling error, and are shown in Figure 12. The dotted line in the figures represent $95 \%$ confidence boundary. It is clearly evident that the auto-correlation and cross-correlation plots for the estimated models were within the $95 \%$ interval, showing that the predicted models optimised by the optimisation algorithms under study were acceptable. Clearly, the auto-correlation and cross-correlation plots of the vertical channel model optimised by HBCSD have satisfied the $95 \%$ boundary better than the other two algorithms. 

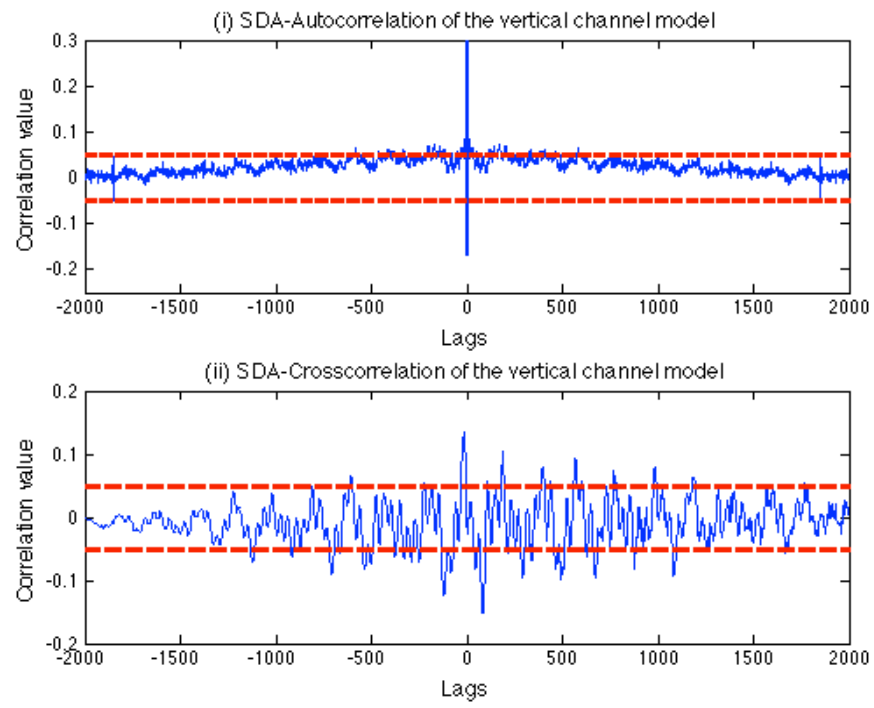

(a) SDA
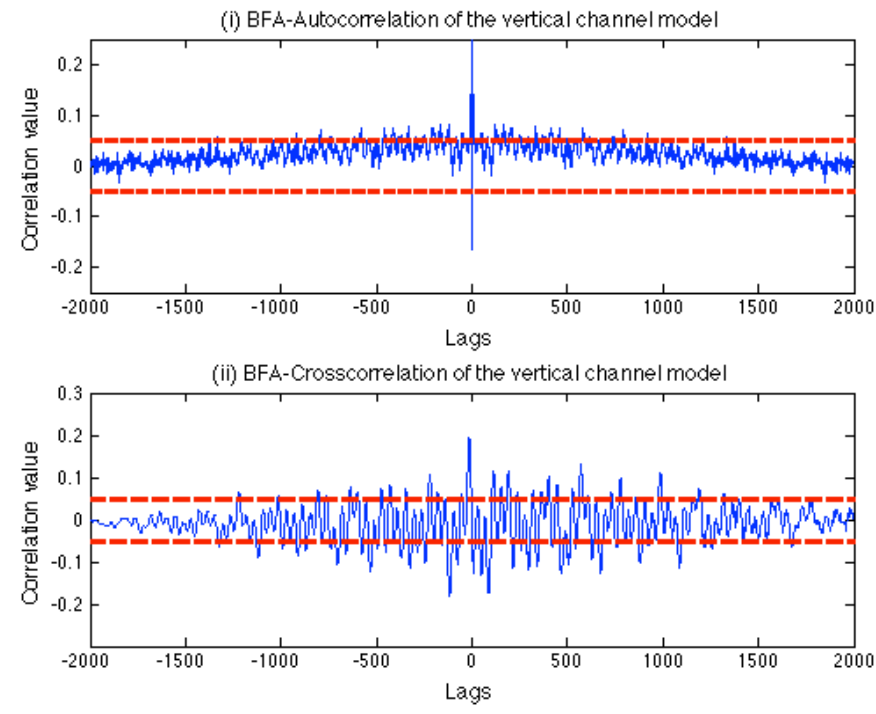

(b) BFA
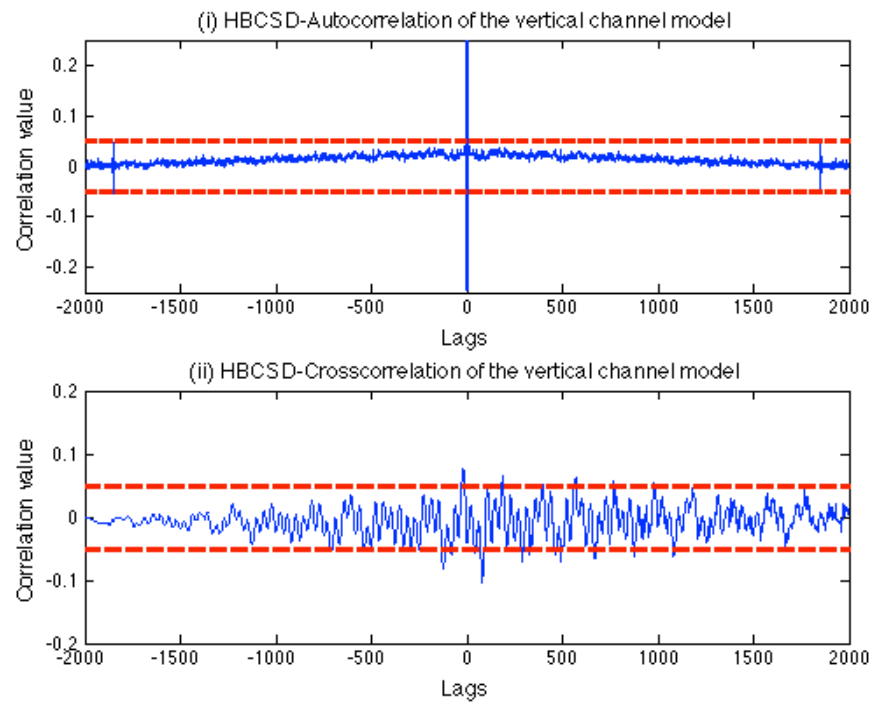

(c) HBCSD

Fig. 12. Autocorrelation and crosscorrelation for the vertical channel model of TRS. 


\section{CONCLUSION}

A novel hybrid bacteria-chemotaxis spiral-dynamics optimisation algorithm namely HBCSD has been proposed. Chemotactic strategy of bacteria through spiral tumble and swim actions of bacteria has been adopted to improve the exploration strategy of SDA. Moreover, spiral radius and angular displacement of the spiral model have been made adaptive to enhance the movement of bacteria within a feasible region. Incorporating these two schemes and excluding the reproduction, elimination and dispersal phases as an alternative to reduce the complexity of BFA has successfully avoided the SDA and BFA from getting trapped into local optima points and has resulted in faster convergence. The proposed algorithm has been utilised to optimise ARX model for flexible systems through linear parametric modelling approach using experimental input and output data from laboratory scale flexible systems. Results have shown that the proposed hybrid algorithm has outperformed its predecessor algorithms in terms of convergence speed and fitness accuracy. Time-domain results have shown that the predicted model response using HBCSD algorithm has successfully followed the actual response. Moreover, frequency-domain results have indicated that the estimated models based on HBCSD algorithm have successfully captured the dynamic behaviours of the flexible systems very well and better than SDA and BFA. The proposed algorithm has effectively optimised the dynamic models of the flexible systems and thus is suitable to use as an optimisation tool for solving real world problems in various applications. In future work, it is envisaged to apply the algorithm to nonlinear modelling of flexible systems using neural networks and fuzzy logic paradigms.

\section{ACKNOWLEDGMENT}

Ahmad Nor Kasruddin Nasir is on study leave from Faculty of Electrical \& Electronics Engineering (FKEE), Universiti Malaysia Pahang (UMP) and is sponsored by Ministry of Higher Education (MOHE), Malaysia.

\section{REFERENCES}

Ahmad, S. M., Chipperfield, A. J., and Tokhi, M. O. (2001). Parametric modeling and dynamic characterization of two-degree-of-freedom twin-rotor multi-input multi-output system. Proceeding of the Institution of Mechanical Engineers, part G: Journal of Aerospace Engineering. 215 (2), pp. 63 - 78.

Alam, M. S. and Tokhi, M. O. (2007a). Dynamic modeling of a single-link flexible manipulator system: a particle swarm optimization approach. Journal of Low Frequency Noise, Vibration and Active Control, $26(1)$, pp. $57-72$.

Alam, M. S. and Tokhi, M. O. (2007b). Modelling of a twin rotor system: a particle swarm optimization approach. Proceeding of the Institution of Mechanical Engineers, part G: Journal of Aerospace Engineering. 221 (3), pp. $353-374$.

Aldebrez, F. M., Mat Darus, I. Z., and Tokhi, M. O. (2004). Dynamic modeling of a twin rotor system in hovering position. Proceeding of the First International Symposium on Control, Communication and Signal Processing, Hammamet Tunisia, March 21- 24, 2004, pp. 823 - 826.

Azad, A. K. M. (1994). Analysis and design of control mechanisms for flexible manipulator systems. PhD Thesis, Department of Automatic Control and Systems Engineering, The University of Sheffield, UK

Billings, S. A. and Voon, W. S. F. (1986). Correlation based validity tests for nonlinear models. International Journal of Control, 44 (1), pp. 235 - 244.

Biswas, A., Dasgupta, S., Das, S., and Abraham, A. (2007a). Synergy of PSO and bacterial foraging optimization-A comparative study on numerical benchmarks. Advances in Soft Computing, (44), pp. 255-263.

Biswas, A., Dasgupta, S., Das, S., and Abraham, A. (2007b). A synergy of differential evolution and bacterial foraging optimization for global optimization, Neural Network, 17 (6), pp. 607-626.

Dasgupta, S., Das, S., Abraham, A., and Biswas, A. (2009). Adaptive computational chemotaxis in bacterial foraging optimization: An analysis, IEEE Transactions on Evolutionary Computation, 13(4), pp. 919941.

Feedback Instruments Ltd. Twin rotor MIMO system manual 33-007-0, Sussex, UK, 1996.

Fujimori, A., Nikiforuk, P. N. and Koda, E. (1995). Modelling of a flexible arm using system identification and model reduction. Journal of Systems and Control Engineering. 209, pp. $13-21$.

Gharooni, S., Heller, B., Tokhi, M.O. (2001). A new hybrid spring orthosis for controlling hip and flexion in the swing phase. IEEE Trans. Neural Syst. Rehabil. Eng., 9 (1), pp. 106 - 107.

$\mathrm{Hu}, \mathrm{Q}$. (2009). Variable structure maneuvering control with time-varying sliding surface and active vibration damping of flexible spacecraft with input saturation, Acta Astronautica, 64 (11-12), pp. 1085 - 1108.

Kim, D. H., Abraham, A., and Cho, J. H. (2007). A hybrid genetic algorithm and bacterial foraging approach for global optimization. Information Sciences, 177(18), pp. 3918-3937.

Liu, K. and Sun, X. (2001). System identification and model reduction for a single-link flexible manipulator. Journal of Sounds and Vibration. 242 (5), pp. $867-891$.

Ljung, L. (1999). System identification: theory for the user 2nd edition. Englewood Cliffs, HJ: Prentice hall. 
Lohokare M. R., Pattnaik S. S., Devi S., Panigrahi B. K.,Das S., Bakwad K. M. (2009). Intelligent biogeography-based optimization for discrete variables. Proceeding of World Congress on Nature \& Biologically Inspired Computing (NaBIC 2009), Coimbatore, India, 9 - 11 December, 2009, pp. 1088 1093.

Majhi, R., Panda, G., Majhi, B., and Sahoo, G. (2009). Efficient prediction of stock market indices using adaptive bacterial foraging optimization (ABFO) and BFO based techniques. Expert Systems with Applications, 36(6), pp. 10097-10104.

Mat Darus, I. Z., Aldebrez, F. M., and Tokhi, M. O. (2004). Parametric modeling of a twin rotor system using genetic algorithm. Proceeding of the First International Symposium on Control, Communication and Signal Processing, Hammamet Tunisia, March 21- 24, 2004, pp. 115 - 118.

Md. Zain, B. A., Tokhi, M. O. and Abd. Latiff, I. (2009a). Dynamic modeling of a single-link flexible manipulator using particle swarm optimization. The Second International Conference on Control, Instrumentation and Mechatronics Engineering (CIM09), Malacca, Malaysia, June 2 - 3, 2009.

Md. Zain, B. A., Tokhi, M. O. and Md. Salleh, S. (2009b). Dynamic modeling of a single-link flexible manipulator using parametric techniques with genetic algorithms. Proceeding of 2009 Third UKSim European Symposium on Computer Modelling and Simulation, Athens, Greece, November 25 - 27 , 2009, pp. $373-378$.

Mishra S., (2005). Hybrid least square-fuzzy bacterial foraging strategy for harmonic estimation. IEEE Transaction on Evolutionary Computation, 9(1), pp. $61-73$.

Nasir, A. N. K., Tokhi, M. O., Abd Ghani, N. M., Ahmad, M. A. (2012a). A novel hybrid spiral dynamics bacterial-chemotaxis algorithm for global optimization with application to controller design. Proceeding of 2012 UKACC International Conference on Control, Cardiff, UK, 3-5 September 2012, pp. 753 - 758.

Nasir, A. N. K., Tokhi, M. O., Abd Ghani, N. M., Ahmad, M. A. (2012b). A novel hybrid spiral dynamics bacterial-foraging algorithm for global optimization with application to control design. Proceeding of 12th Annual Workshop on Computational Intelligence (UKCI2012), Edinburgh, UK, 5-7, September 2012, pp. 1-7.

Nasir, A. N. K., Tokhi, M. O., Abd Ghani, N. M. and Raja Ismail, R. M. T. (2012c). Novel adaptive spiral dynamics algorithms for global optimization. Proceeding of $11^{\text {th }}$ IEEE International Conference on Cybernatic Intelligent System 2012, Limerick, Ireland, 23-24 August 2012, pp. 99-104.

Nasir, A. N. K., and Tokhi, M. O. (2013a). A novel hybrid spiral-dynamics random-chemotaxis optimization algorithm with application to modelling of a flexible robot manipulator. Proceeding of 2013 16th International Conference on Climbing and Walking Robots (CLAWAR 2013), Sydney, Australia, July 14-17, 2013, pp. 667-674.

Nasir, A. N. K., Tokhi, M. O., Ghani, N. M. A. (2013b). Novel hybrid bacteria foraging and spiral dynamics algorithms. Proceeding of 13th Annual Workshop on Computational Intelligence (UKCI2013), Surrey, UK, 9-11, September 2013, pp. 199-205.

Neri, F., and Cotta, C. (2012). Memetic algorithms and memetic computing optimization: A literature review. Swarm and Evolutionary Computation, 2, pp. 1-14.

Niño, J., Mitrache, F., Cosyn, P., and Keyser, R. De. (2007). Model Identification of a Micro Air Vehicle, Journal of Bionic Engineering 4, pp. 227-236.

Omar, M., Zaidan, M. A. and Tokhi, M. O. (2011). Dynamic modelling and control of a twin-rotor system using adaptive neuro-fuzzy inference system techniques. Proceeding of Institution of Mechanical Engineers, Part G: Journal of Aerospace Engineering, 226 (7), pp. 787-803.

Osman, I. B. and Laporte, G. (1996). Metaheuristics: A bibliography. Annals of Operations Research, 63 (5), pp. 511-623

Ostergaard, E.H., (2012). Lightweight robot for everybody. IEEE Robotics \& Automation Magazine, 19 (4), pp. $17-18$.

Panigrahi, B. K., and Ravikumar, P. V., (2008). Bacterial foraging optimisation: Nelder - Mead hybrid algorithm for economic load dispatch, IET Generation, Transmission \& Distribution, 2(4), pp. 556-565.

Panikhom, S., Sarasiri, N., and Sujitjorn, S. (2010). Hybrid Bacterial Foraging and Tabu Search Optimization (BTSO) Algorithms for Lyapunov's Stability Analysis of Nonlinear Systems, International Journal of Mathematics and Computers in Simulation, 4(3), pp. 81-89.

Passino, K. M. (2002). "Biomimicry of bacterial foraging for distributed optimization and control," IEEE Control System Magazine, June 2002, pp. $52-67$.

Raptis, I. A., Valavanis, K. P. and Vachtsevanos, G. J. (2012). Linear tracking control for small-scale unmanned helicopters. IEEE Transactions On Control Systems Technology, 20 (4), pp. $995-1010$.

Rovner, D. M. and Cannon, Jr. R. H. (1987). Experiments towards on-line identification and control of a very flexible one-link manipulator. The International Journal of Robotics Research. 6, pp. 3-19.

Shaheed, M. H. and Tokhi, M. O. (2002). Dynamics modelling of a single-link flexible manipulator: parametric and non-parametric approaches. Robotica. 20 (1), pp. 93 - 109.

Sjoberg, J., Zhang, Q., Ljung, L., Benveniste, A., Delyon, B., Glorennec, P. Y., Hjalmarsson, H., and Juditsky A. (1995). Nonlinear black-box modeling in system identification: Unified overview, Automatica, 31(12), pp. 1691-1724.

Subudhi, B. and Jena, D. Nonlinear system identification of a twin rotor MIMO system. Proceedings of the IEEE TENCON 2009 Conference, Singapore, 23-26 November 2009, pp. 1-6. 
Supriyono, H. and Tokhi, M. O. (2012). Parametric modeling approach using bacterial foraging algorithms for modeling of flexible manipulator systems. Engineering Application and Artificial Intelligence Systems. 25 (5), pp. $898-916$.

Tamura, K., and Yasuda, K. (2011a). Primary study of spiral dynamics inspired optimization. IEEJ Transactions on Electrical and Electronic Engineering, 6 (S1), pp. 98 - 100.

Tamura, K., and Yasuda, K. (2011b). Spiral multipoint search for global optimization. Proceeding of $201110^{\text {th }}$ International Conference on Machine Learning and Applications, Honolulu, HI, 18-21 Dec 2011, pp. $470-475$.

Tavakolpour, A. R., Mat Darus, I. Z., Tokhi, O., and Mailah, M. (2010). Genetic algorithm-based identification of transfer function parameters for a rectangular flexible plate system. Engineering Applications of Artificial Intelligence, 23(8), pp. 1388-1397.

Toha, S. F. and Tokhi, M. O. (2010). Parametric modeling application to a twin rotor system using recursive least square, genetic and swarm optimization techniques. Proceeding of the Institution of Mechanical Engineers, part G: Journal of Aerospace Engineering. 224, pp. $961-977$.

Toha, S. F., Julai, S. and Tokhi, M. O. (2012). Ant colony based model prediction of a twin rotor system. Procedia Engineering. 41, pp. $1135-1144$.

Tokhi, M.O., Azad, A.K.M. (1995). Real-time finite difference simulation of a single-link flexible manipulator system incorporating hub inertia and payload. Proceedings of the Institution of Mechanical Engineers. Part I, Journal of systems and control engineering, 209 (1), pp. 21-33.

Tokhi, M.O., Mohamed, Z. (1999). Finite element approach to dynamic modelling of a flexible robot manipulator: Performance evaluation and computational requirements. Communications in Numerical Methods in Engineering, 15 (9), pp. 669-678.

Tokhi, M.O., Mohamed, Z., Amin, S.H.M., Mamat, R. (2000). Dynamic characterization of a flexible manipulator system : theory and experiments. Proceeding of TENCON 2000, Kuala lumpur Malaysia, 24 - 27 Sept 2000, pp. 167-172.

Torsten, S. and Petre, S. (2001). System Identification. Prentice Hall International (UK) Ltd, International Series in Systems and Control Engineering.

Venkaiah, C., and Vinod Kumar, D. M. (2011). Fuzzy adaptive bacterial foraging congestion management using sensitivity based optimal active power re-scheduling of generators. Applied Soft Computing, 11(8), pp. 4921-4930.

Yatim, H. M., Mat Darus, I. Z. and Mohamad, M. (2012). Parametric identification and dynamic characterization of flexible manipulator system. Proceeding of 2012 IEEE Conference on Control, Systems and Industrial Informatics, Bandung Indonesia, 23-26 Sept. 2012, pp. 16-21.

Yatim, H., Mat Darus, I. Z. and Hadi, M. S. (2013). Particle swarm optimization for identification of a flexible manipulator system. Proceeding of the 2013 IEEE Symposium on Computers and Informatics, Langkawi, Malaysia, 7-9 April 2013, pp. 112-177.

Yurkovich, S., Tzes, A. P., Lee, I. and Jillsley, K. L. (1990). Control and system identification of a two-link flexible manipulator. Proceedings of the IEEE International Conference Robotics and Automation, Cincinnati, OH, pp. $1626-1631$.

Zang, H., Zhang, S., and Hapeshi, K. (2010). A Review of Nature-Inspired Algorithms. Journal of Bionic Engineering, 7, pp. S232-S237.

Zhong, Y., Lin, J., Ning, J., and Lin, X. (2011). Hybrid artificial bee colony algorithm with chemotaxis behavior of bacterial foraging optimization algorithm. Proceeding of 2011 Seventh International Conference on Natural Computation, Shanghai, 26-28 July 2011, pp.1171-1174. 